INRA Prod. Anim.,

2013, 26 (2), 207-220

\title{
Impact environnemental des systèmes bovins laitiers français
}

\author{
J.-B. DOLLE' ${ }^{1}$, L. DELABY $Y^{2,3}$, S. PLANTUREUX ${ }^{4,5}$, S. MOREAU ${ }^{6}$, B. AMIAUD ${ }^{10,11}$, A. CHARPIOT \\ V. MANNEVILLE ${ }^{8}$, A. CHANSEAUME ${ }^{4,5,8}$, H. CHAMBAUT', A. LE GALL \\ ${ }^{1}$ Institut de l'Elevage, BP 80039, F-62051 Saint-Laurent-Blangy, France \\ 2 INRA UMR1348 PEGASE, F-35590 Saint-Gilles, France \\ 3 Agrocampus Ouest, UMR1348 PEGASE, F-35000 Rennes, France \\ 4 Université de Lorraine, UMR1121 LAE, F-54505 Vandøuvre-lès-Nancy, France \\ 5 INRA, UMR1121 LAE, F-54505 Vandouvre-lès-Nancy, France \\ 6 Institut de l'Elevage, F-69364 Lyon, France \\ ${ }^{7}$ Institut de l'Elevage, BP 85225, F-35652 Le Rheu, France \\ 8 Institut de l'Elevage, F-63170 Aubière, France \\ ${ }^{9}$ Institut de l'Elevage, BP 70510, F-49105 Angers, France \\ 10 INRA, UMR1137 EEF, F-54280 Champenoux, France \\ 11 Université de Lorraine, UMR1137 EEF, F- 54506 Vandouvre-lès-Nancy, France
}

Courriel : jean-baptiste.dolle@idele.fr

L'élevage laitier doit à la fois produire plus pour répondre au défi alimentaire mondial mais également produire différemment en assurant la préservation de l'environnement. Cet article fait le point sur l'impact environnemental des systèmes d'élevage laitiers vis-à-vis de la préservation de la qualité de l'eau, de la réduction des émissions gazeuses et du maintien de la biodiversité.

Les exploitations bovines laitières, qui occupent près de la moitié de la surface agricole française $(46 \%)$, ont un rôle extrêmement important à jouer en France vis-à-vis de l'environnement. Avec 76648 fermes laitières en 2010 (source Recensement Agricole 2010) soit $16 \%$ des exploitations françaises pour une collecte laitière nationale de 24 milliards de litres (CNIEL 2012), la production laitière a connu de fortes restructurations depuis l'instauration des quotas laitiers. La diminution importante du nombre de vaches en 20 ans (5,3 millions de vaches laitières en 1990 et 3,7 millions en 2010), accompagnée d'une augmentation du rendement par vache (respectivement 4000 et 6400 litres par vache) (CNIEL 2012), a conduit à une spécialisation et à une intensification des systèmes de production. Cette intensification, qui concerne les cultures fourragères et l'animal, a souvent été permise par un recours aux engrais minéraux et aux aliments du bétail, notamment sur la période 1990 2010 et s'est traduite par des excédents azotés à l'échelle du système de production (Le Gall et al 2005, Raison et al 2008).

Ces excédents azotés et les risques environnementaux associés dépendent cependant assez fortement de la zone de production et du niveau d'intensification. Deux situations contrastées coexistent ainsi en France et présentent des niveaux d'intensification fourragère et animale différenciés (Le Gall et al 2009). Les régions laitières de montagne ou semimontagne d'Auvergne et Franche-Comté et les zones herbagères de l'Est de la France, qui possèdent une forte proportion de prairies permanentes, une faible part de maïs ensilage et des chargements modérés, enregistrent de faibles excédents azotés, mais une forte dépendance énergétique du fait d'un recours important aux concentrés. Inversement, les régions de cultures fourragères de l'Arc Atlantique et des pourtours du Massif Central, qui présentent une part importante de maïs ensilage et de prairies temporaires ainsi qu'une intensification laitière importante permise par une utilisation d'engrais et de concentrés, occasionnent des excédents d'azote plus élevés quelle que soit la zone. Globalement, l'alimentation des vaches laitières et des génisses d'élevage repose essentiellement sur les fourrages, qui représentent 70 à $100 \%$ de la ration annuelle des animaux (Devun et al 2012). Ces fourrages, produits à plus de 90\% sur la Surface Agricole Utile (SAU) des exploitations (Paccard et al 2003), leur confèrent une forte autonomie fourragère. En conséquence, les exploitations laitières disposent dans leur grande majorité d'une capacité de valorisation des déjections animales importante qui limite les risques environnementaux. Ce recyclage n'est toutefois pas total car des pertes d'azote (figure 1), de phosphore et de carbone se produisent à chaque étape de cette valorisation, que ce soit sur la parcelle ou en stabulation. L'intensification laitière peut également contribuer à l'altération des équilibres des écosystémes ainsi qu'à la simplification des paysages par la suppression des milieux semi-naturels comme les bois, les haies, les prairies permanentes (Le Roux et al 2008). Néanmoins, l'élevage laitier dispose aussi de réels atouts parce qu'il est directement gestionnaire de surfaces, de milieux et d'infrastructures agro-écologiques importantes, favorables au stockage de carbone et à la biodiversité, qui fournissent à la collectivité de nombreux services écosystémiques et environnementaux (Chevassus-au-Louis et al 2009).

Cet article vise à apporter un éclairage sur les pertes relatives aux éléments minéraux $(\mathrm{N}, \mathrm{P} \ldots)$, qui se produisent vers l'eau avec pour conséquence l'altération de la qualité des nappes phréatiques, des cours d'eau et la contribution au développement des algues vertes en zones côtières sensibles; ainsi que les pertes d'azote et de carbone vers l'air 
Figure 1. Flux et pertes d'azote à l'échelle de l'exploitation (adapté de Jarvis et al 2011 et Gac et al 2007).

Exemple d'une exploitation lait + céréales (80 ha, 82 UGB, 25 ha céréales).

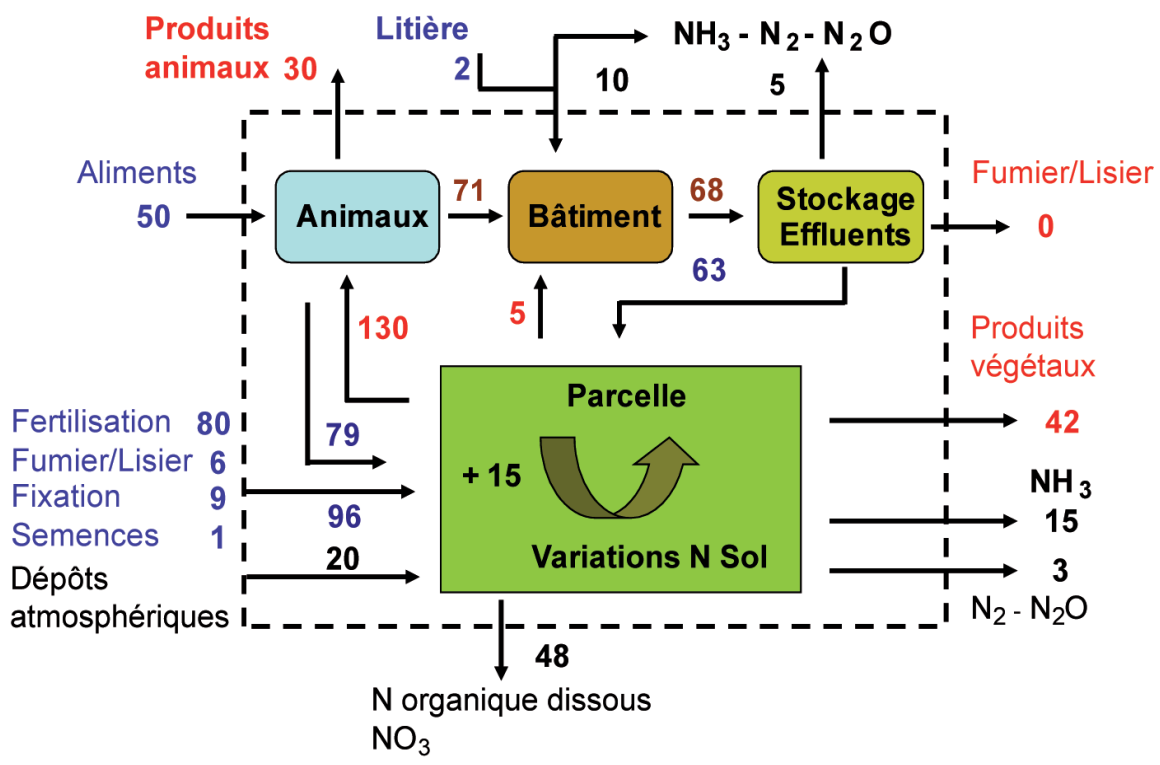

qui concernent les Gaz à Effets de Serre (GES), responsables du changement climatique $\left(\mathrm{N}_{2} \mathrm{O}\right.$ et $\left.\mathrm{CH}_{4}\right)$, et l'ammoniac $\left(\mathrm{NH}_{3}\right)$, responsable de l'acidification du milieu et de la production de particules dangereuses pour la santé humaine. Un volet spécifique précisera également le rôle joué par l'élevage laitier sur le maintien de la biodiversité. Une dernière partie sera consacrée à l'évaluation des impacts potentiels sur l'air et sur l'eau, et de la contribution à la biodiversité des principaux systèmes laitiers nationaux.

\section{1 / Elevage laitier et qualité de l'eau}

Pour diverses raisons, telle l'existence de la réglementation européenne (Directive Nitrates, cf. encadré 1) et le rôle pilote de l'azote dans les systèmes d'élevage et agricoles, nous nous intéresserons surtout aux flux d'azote et dans une moindre mesure aux flux de phosphore associés aux activités d'élevage laitier. Il importe alors de mieux décrire et comprendre l'origine et les quantités associées à ces flux afin d'en limiter les pertes et en accroître le recyclage et la valorisation, ceci dans le but d'améliorer les performances tant économiques qu'écologiques des systèmes d'élevage.

\section{1 / Les flux à l'échelle de l'animal}

Les principaux facteurs de variation de l'excrétion d'azote $(\mathrm{N})$ et de phosphore $(\mathrm{P})$ chez la vache laitière et les génisses d'élevage sont souvent décrits et quantifiés (Delaby et al 1995, Micol et al 2003) en s'appuyant sur la simple équation:

Qtés excrétées $(\mathrm{g} / \mathrm{j})=$ Qtés ingérées Qtés fixées (lait, croissance) qualité de l'eau. la pollution par les nitrates d'origine agricole. applicables aux élevages bovins depuis 1992. gramme. animale, etc.
Lucbert 1999, Dulphy et Grenet 2001). Les restitutions totales annuelles d'une vache laitière produisant $6000 \mathrm{~kg}$ de lait varient entre 80 et $122 \mathrm{~kg}$ d'N et 16 et $18 \mathrm{~kg}$ de P (Delaby et Lucbert 1999 , Vérité et Delaby 2000) selon la part de maïs et d'herbe qui compose la ration. Compte tenu de la richesse en $\mathrm{N}$ de l'herbe pâturée notamment au printemps et à l'automne et de la présence de légumineuses, l'excrétion d'azote est d'autant plus importante que la part de l'herbe dans la ration est importante.

Si l'excrétion de phosphore est réalisée pour l'essentiel par voie fécale (Meschy 2010), l'excrétion d'azote se produit à la fois par les voies urinaire et fécale. La partie fécale, qui correspond à la fraction de l'azote indigestible des aliments et des microbes ainsi qu'à l'azote endogène (Peyraud et al 1995), est associée directement aux quantités de MS ingérées avec un coefficient d'excrétion qui varie entre 7 et $8 \mathrm{~g} / \mathrm{kg}$ de MS ingérée (Demarquilly et al 1995). Toutefois pour certaines rations comportant des plantes riches en tanins (sainfoin par exemple), cette relation directe entre l'ingestion et l'excrétion fécale est modifiée avec une augmentation sensible de l'N fécal excrété (Aufrère et al 2012). L'azote fécal est sous forme organique et peu disponible. Il présente donc en l'état de faibles risques environnementaux. L'N urinaire, qui représente l'exutoire naturel des excédents, est excrété surtout sous forme d'urée. Rapidement minéra-

Encadré 1. Règlementation environnementale en relation avec la préservation de la

La Directive européenne $n^{\circ}$ 91/676/CEE du 12 décembre 1991 couramment appelée Directive Nitrates, vise la lutte contre la pollution par les nitrates d'origine agricole. Cette Directive indique les principes à mettre en œuvre pour assurer la protection des eaux contre

En France, le code des bonnes pratiques (Arrêté du 22 novembre 1993) fixe les exigences techniques en matière de stockage et d'épandage des déjections animales afin de réduire les pollutions ponctuelles et diffuses. Le dispositif juridique français comprend également une réglementation concernant les Installations Classées pour la Protection de l'Environnement (ICPE) qui fixe des prescriptions techniques en fonction de la taille des élevages,

Dans ce contexte, les pouvoirs publics et la profession agricole ont décidé conjointement, en 1994, de mettre en place un Programme de Maîtrise des Pollutions d'Origine Agricole, appelé PMPOA I, axé sur la mise aux normes des bâtiments des exploitations agricoles et la maîtrise de la fertilisation minérale et organique. Un PMPOA II, ciblant uniquement les élevages situés dans les zones vulnérables au sens de la Directive Nitrates et la promotion des actions en faveur de la préservation de la qualité de l'eau, a prolongé ce précédent pro-

Au terme de ces deux programmes ambitieux, le 20 novembre 2009, la France fait néanmoins l'objet d'une mise en demeure de la part de la Commission Européenne pour mauvaise application de la Directive Nitrates. Cette mise en demeure porte sur le dispositif réglementaire français fixant le cadre national des programmes d'actions, jugé peu ambitieux par la Commission Européenne. En réponse à la mise en demeure européenne, l'état français publie le 19 décembre 2011 un nouvel arrêté relatif au programme d'actions national à mettre en œuvre dans les zones vulnérables. Cet arrêté fixe de nouvelles périodes d'interdiction d'épandage, de nouvelles prescriptions pour le stockage des effluents, la limitation de l'épandage des fertilisants, de nouvelles normes d'excrétion azotée par catégorie 
lisable et plus disponible, elle présente plus de risques vis-à-vis des pertes vers l'eau ou vers l'air tout au long du cycle de valorisation. Tout excès alimentaire, tant au niveau de l'animal (apports besoins) qu'au niveau du rumen (PDIN - PDIE) se traduit par une augmentation des rejets d'azote dans l'urine (Peyraud et al 1995, Calsamiglia et al 2010). La distinction entre excrétion fécale et urinaire n'a que peu d'intérêt lorsque l'on s'intéresse aux effluents stockés (fumier, lisier...) alors qu'elle prend toute son importance au pâturage. En effet, au pâturage, les restitutions sont distinctes, très localisées et s'appliquent sur un couvert végétal en croissance.

\section{2 / Les flux à l'échelle de l'atelier laitier}

A l'échelle de l'atelier laitier, les pertes d'azote et de phosphore sont liées au bilan entre les restitutions via les effluents épandus, les restitutions animales au pâturage et les prélèvements par les plantes. Ainsi, selon Peyraud et al (1995), l'augmentation de la part d'herbe dans le régime annuel d'un troupeau de vaches laitières se traduit par une augmentation des rejets totaux d'azote, mais également par une augmentation des capacités de valorisation et d'exportation de l'N par les cultures associées (tableau 1). Le bilan apparent des minéraux (Simon et Le Corre 1992), qui permet de quantifier les flux d'azote ou de phosphore qui entrent et sortent de l'exploitation via les achats et/ou les cessions et donc de connaître l'azote qui restera sur l'exploitation, est un bon indicateur de gestion de l'azote et des risques très globaux vis-à-vis de l'air et de l'eau (Schröder et al 2003). Compte tenu des importations d'aliments et d'éléments fertilisants exogènes, la pression animale par hectare peut excéder les capacités de production du milieu et altérer son équilibre (Peyraud et al 2012). Les excédents d'azote issus des calculs de bilans apparents des fermes de l'Arc Atlantique sont aujourd'hui compris entre 90 et $150 \mathrm{~kg}$ par hectare, alors qu'ils étaient de l'ordre de 150 à $250 \mathrm{~kg} \mathrm{~N} / \mathrm{ha}$ dans les années 1980-1990 (tableau 2). Afin de poursuivre la réduction des excédents d'azote, il faut agir principalement sur les entrées par les engrais et les aliments du bétail, en augmentant le recyclage interne des déjections animales. Au regard des premiers bilans apparents réalisés en Bretagne, les résultats obtenus dans les fermes laitières de l'ouest de la France montrent que le raisonnement de la fertilisation, le remplacement des graminées pures très fertilisées par des associations ray-grass anglais trèfle blanc ainsi que la réduction des apports de concentrés permettent de réduire les excédents de façon importante.

Tableau 1. Effet du système fourrager sur les rejets azotés des animaux et la capacité de valorisation des cultures associées (Peyraud et al 1995).

\begin{tabular}{|l|c|c|c|}
\hline & $\begin{array}{c}\text { Ensilage maïs } \\
\text { 12 } \text { mois }^{(1)}\end{array}$ & $\begin{array}{c}\text { Ensilage maïs + } \\
\text { pâturage 3 } \text { mois }^{(2)}\end{array}$ & $\begin{array}{c}\text { Ensilage (maïs + herbe) + } \\
\text { pâturage 6 mois }\end{array}$ \\
\hline Rejets azotés totaux (kg) & 3800 & 4250 & 5100 \\
\hline $\begin{array}{l}\text { Rejets azotés totaux } \\
\text { (sans aucune perte, kg/ha) }\end{array}$ & 190 & 190 & 230 \\
\hline $\begin{array}{l}\text { Exportations d'N par les } \\
\text { cultures (kg/ha) }\end{array}$ & 160 & 180 & 240 \\
\hline
\end{tabular}

cultures $(\mathrm{kg} / \mathrm{ha})$

(1) Troupeau de 40 vaches (7500 kg lait/vache) et 20 ha de SAU cultivés en ensilage de maïs.

(2) Même troupeau et 22,5 ha SAU dont 8 ha de ray-grass anglais.

(3) Même troupeau et 22,5 ha SAU dont 16 ha de ray-grass anglais ( 8 ha récoltés en ensilage).

Tableau 2. Evolution des excédents d'azote dans les exploitations laitières de Bretagne et des Pays-de-la-Loire.

\begin{tabular}{|c|c|c|c|c|c|}
\hline Régions & $\begin{array}{c}\text { Bretagne et } \\
\text { Pays de la Loire }\end{array}$ & \multicolumn{2}{|c|}{ Bretagne } & \multicolumn{2}{|c|}{$\begin{array}{c}\text { Bretagne et } \\
\text { Pays de la Loire }\end{array}$} \\
\hline Système & Lait & Lait & $\begin{array}{l}\text { Lait et } \\
\text { hors sol }\end{array}$ & Lait & $\begin{array}{l}\text { Lait et } \\
\text { hors sol }\end{array}$ \\
\hline Années d'études & 1989-1994 & \multicolumn{2}{|c|}{$1995-1996$} & \multicolumn{2}{|c|}{$2000-2005$} \\
\hline Nombre d'exploitations* & 48 & 128 & 11 & 27 & 7 \\
\hline Source & SIMON et al 2000 & \multicolumn{2}{|c|}{ LE GALL 2005} & \multicolumn{2}{|c|}{$\begin{array}{c}\text { RESEAUX D'ELEVAGE } \\
\text { BOVINS LAIT }\end{array}$} \\
\hline Chargement (UGB/ha SFP) & 1,8 & 1,8 & 1,8 & 1,8 & 1,6 \\
\hline Maïs (\% de la SFP) & 46 & 33 & 28 & 39 & 30 \\
\hline Lait produit (kg/ha SAU) & 6400 & 5650 & 5800 & 5000 & 4100 \\
\hline Cultures (\% de la SAU) & 12 & 19 & 15 & 25 & 31 \\
\hline Entrées (kg N/ha SAU) ${ }^{* *}$ & 276 & 196 & 471 & 155 & 250 \\
\hline dont fertilisation minérale & 200 & 100 & 101 & 78 & 60 \\
\hline dont concentrés & 72 & 49 & 327 & 43 & 170 \\
\hline Sorties (kg N/ha SAU) & 59 & 54 & 180 & 63 & 99 \\
\hline Excédent du bilan (kg N/ha SAU) & 217 & 142 & 291 & 92 & 151 \\
\hline Taux efficacité sorties/entrées (\%) & 21 & 27 & 38 & 40 & 39 \\
\hline
\end{tabular}

* Nombre d'exploitations moyen par an.

${ }^{* *}$ Les entrées sont composées des postes fertilisation minérale, fixation symbiotique, importation de déjections et aliments achetés (fourrages et concentrés).

A l'échelle de l'exploitation, l'évaluation des risques de lixiviation consiste à mettre en regard ces excédents azotés du bilan apparent à la lame drainante, tout en intégrant la notion de risques associés au système de cultures en place sur l'exploitation (\% herbe dans l'assolement, cultures annuelles, couvert végétal d'automne). Ainsi, Raison et al (2008) montrent que, pour des lames drainantes de $400 \mathrm{~mm}$ ou plus, les pertes d'azote par lixiviation sont comprises entre 30 et $60 \mathrm{~kg} / \mathrm{ha} / \mathrm{an}$ pour des excédents d'azote de 80 à $150 \mathrm{~kg} / \mathrm{ha} / \mathrm{an}$ dans les systèmes de cultures fourragères de l'ouest de la France. Elles sont seulement de 10 à $20 \mathrm{~kg} / \mathrm{ha} / \mathrm{an}$ dans les systèmes herbagers d'Irlande et du Royaume-Uni, basés sur des prairies de longue durée, alors que les excédents d'azote y sont 1,5 à 2 fois plus élevés (figure 2). Les systèmes de cultures fourragères annuelles, avec des ruptures dans la couverture des sols, exigent ainsi une grande maîtrise technique (limitation des entrées d'azote, recyclage des engrais de ferme, cultures intermédiaires...) pour réduire les pertes d'azote nitrique et, atteindre les objectifs de qualité de l'eau (Aarts et al 2000). Ce d'autant plus que les systèmes d'alimentation qui reposent sur l'ensilage de maïs (20 à 35\% de la Surface Fourragère Principale - SFP), plante certes riche en énergie et à rendement stable, possèdent des capacités limitées à valoriser l'azote (Carpentier et Gabon 2011). Pour les systèmes pâturant, le risque de lessivage est surtout associé à la restitution des déjections au pâturage, notamment du fait des restitutions urinaires localisées. En effet, l'espace de diffusion d'un pissat représente une surface d'environ $1 \mathrm{~m}^{2}$, ce qui se traduit par un apport localisé d'azote qui varie entre l'équivalent de 250 et $300 \mathrm{~kg}$ par hectare, mais peut atteindre l'équivalent de $1000 \mathrm{~kg}$ (Vertès et al 1997). Le lessivage de l'azote urinaire est par ailleurs très dépendant du drainage automnal, mais également de la saison de dépôt du pissat (Vertès et al 1997). Ainsi selon Decau et al $(2003,2004), 15$ à $20 \%$ de l'N urinaire d'un pissat de septembre est lessivé durant l'hiver qui suit, tandis 
Figure 2. Pertes d'azote dans différents systèmes laitiers européens.

Les triangles et cercles blancs représentent les fermes expérimentales suivies dans le projet européen Green Dairy.

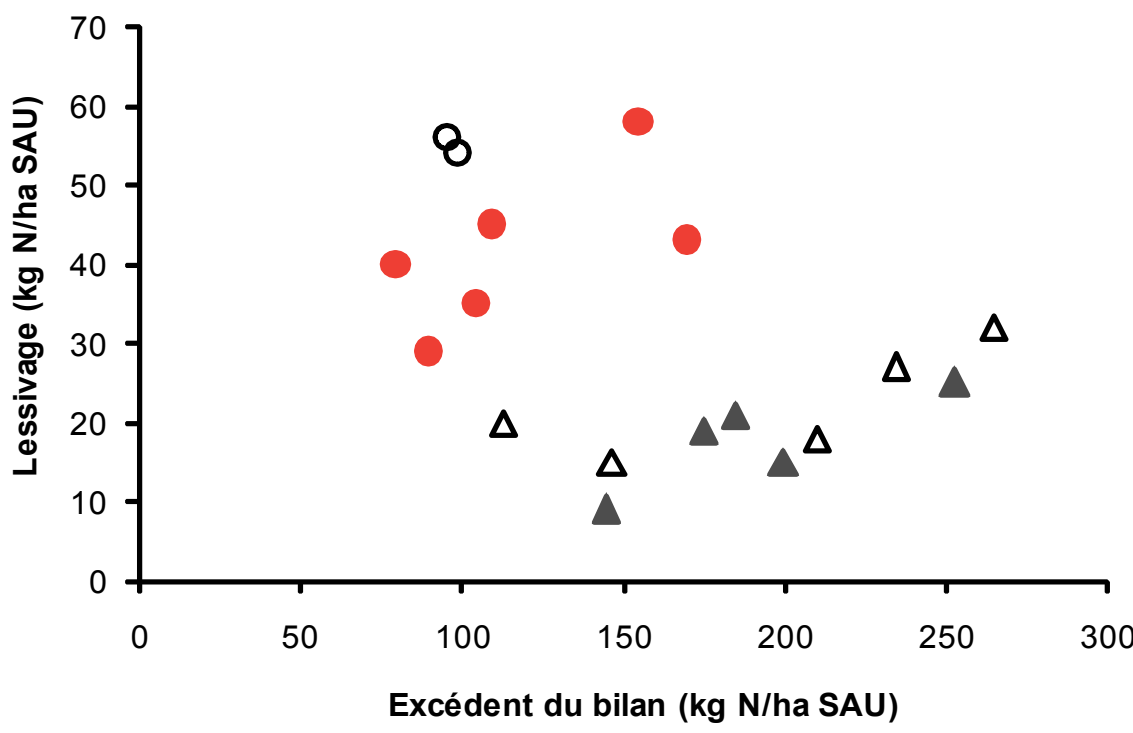

Systèmes de cultures fourragères (France et Pays-Bas)

$\triangle$ Systèmes herbagers (Iles Britanniques)

que seulement 1 à $5 \%$ de l'azote d'un pissat de mars sera perdu et $53 \%$ de l'azote aura pu être valorisé par la plante au cours de l'année. Au niveau des parcelles pâturées, le nombre de jours de pâturage réalisés, qui augmente entre autre avec le niveau de fertilisation azotée, est un excellent indicateur du risque de lessivage (Decau et al 1997, Vertès et al 2007). A ce titre, la pratique de l'alternance fauche/pâture sur l'ensemble de la sole prairiale est préférable à la spécialisation des parcelles (Laurent et al 2000), notamment celles à proximité de l'exploitation dont le nombre de jours de présence des animaux très élevé tend à en faire des parcelles «parking » (Vertès et al 2010). Lors de la collecte des déjections en bâtiment, il n'en reste pas moins vrai que la valorisation des effluents d'élevage reste parfois problématique (risques sanitaires, période d'épandage, aptitude de la culture, topographie des parcelles...), ce qui crée des transferts de fertilité et de possibles dommages environnementaux souvent différés compte tenu des formes d'azote épandues, des temps de transfert et des délais de réaction du milieu (Chevassus-au-Louis et al 2012).

Au-delà des raisonnements à l'échelle du système, il importe de prendre en compte les successions culturales (rotations) et la répartition territoriale des cultures et des zones d'épuration possibles (zones humides, bois, prairies permanentes...) pour évaluer les risques de pollution par les nitrates à l'échelle concernée, à savoir celle du bassin versant (Mignolet et al 1997, Peyraud et al 2012). La démarche agronomique « Territ'eau » proposée dans le cadre de
l'Agrotransfert Bretagne constitue à ce titre une approche intéressante en matière d'évaluation des risques de lessivage qui intègre à la fois cultures, pratiques et rotations (Vertès et al 2010). Plus largement, le couplage de modèles à l'échelle de l'exploitation et du bassin versant (Moreau et al 2013) devrait permettre dans un futur proche de décrire l'impact de changement de pratiques (fertilisation, cultures intermédiaires, répartition des effluents) ou de systèmes d'élevage (chargement, part d'herbe et de maïs dans l'assolement) sur les pertes par lessivage à l'échelle d'un bassin versant et éclairer par exemple les décisions publiques dans le cadre de plans d'action ciblés sur certains territoires.

\section{2 / Elevage laitier et qualité de l'air}

Responsable de $6 \%$ des émissions nationales de GES et de 30\% des émissions d'ammoniac (CITEPA 2010), l'élevage laitier doit intégrer ces problématiques dans le fonctionnement des exploitations pour contribuer à satisfaire les objectifs de réduction des émissions gazeuses assignés à l'agriculture (cf. encadré 2).

\section{1 / Les flux à l'échelle de l'animal}

Les émissions de gaz carbonique et d'eau liées à la respiration des animaux sont biogéniques et ne constituent pas une contribution additionnelle à l'effet de serre. En revanche, le méthane entérique dont la production représente une perte énergétique comprise entre 2 et $12 \%$ de l'énergie ingérée par l'animal (Johnson et Johnson 1995) est anthropique. Le facteur d'émission varie de 90 à $163 \mathrm{~kg}$ de méthane entérique par vache laitière et par an lorsque la production laitière passe de 3500 à $11000 \mathrm{~kg}$ par an. Il est en moyenne de $118 \mathrm{~kg}$ pour une production laitière moyenne en France, évaluée à $6300 \mathrm{~kg}$ par an (Vermorel et al 2008). Sa production est liée à un ensemble de facteurs alimentaires (teneur en matière organique digestible, niveau d'alimentation, qualité du fourrage, quantité de concentré, apport de lipides...) agissant sur les fermentations entériques (Sauvant et al 2011). Parmi les stratégies identifiées pour réduire la production de méthane, l'augmentation de la part de concentrés dans la ration, qui remplace les glucides des parois végétales des fourrages par de l'amidon et des sucres est souvent citée (Doreau et Dollé 2011, Martin et al 2011). Néanmoins ces effets peuvent être contrebalancés par les émissions de GES liées à la production de concentrés utilisés (Doreau et Dollé 2011). Parfois remis en cause par certains auteurs (Woodward et al 2006), un autre levier pour réduire les émissions de méthane entérique concerne l'enrichissement de la ration en lipides insaturés, qui permet une diminution moyenne de $3,8 \%$ des émissions de méthane pour $1 \%$ de lipide supplémentaire dans la ration (Martin et al 2011). Les additifs alimentaires (ionophores, acides organiques) pourraient également constituer une autre voie de réduction des émissions de méthane entérique, cependant leur développement est encore sujet à de nombreuses interrogations (Doreau et Dollé 2011).

Par ailleurs, le niveau de rejets azotés des animaux et la partition de l'azote entre les urines et les fèces influent sur les risques de pertes azotées par voie gazeuse lors de la phase de gestion des déjections.

\section{2 / Les flux à l'échelle de l'atelier}

Comme pour la réduction des pertes azotées vers l'eau, l'optimisation du bilan azoté reste le meilleur moyen de réduire les pertes gazeuses azotées. Au-delà du bilan azoté optimisé, certaines sources d'azote comme les légumineuses constituent également une piste de réduction voire de suppression des émissions de protoxyde d'azote. La gestion des déjections (pâturage vs bâtiment, type de déjection, modalités de stockage et d'épandage...) constitue ensuite l'élément déterminant vis-à-vis des émissions gazeuses azotées et carbonées. Le pâturage, qui évite le transit des déjections par les trois postes que sont le bâtiment, le stockage et l'épandage, représente pour cela la solution la moins émettrice d'azote (Barré 2001, Bolan et al 2004), 
Encadré 2. Règlement environnementale en relation avec la réduction des émissions de GES et d'ammoniac.

Pour réduire l'effet des émissions de Gaz à Effet de Serre (GES) sur le changement climatique, le protocole de Kyoto, ratifié en 2005, visait une réduction des émissions de GES au niveau international de 5,2\% en 2012 par rapport à 1990. Dans le cadre d'un second engagement post 2012 , les objectifs de réduction pourraient être portés à $20 \%$ voire $30 \%$ d'ici 2020 par rapport à 1990. Suite au conseil européen de mars 2007, la décision $n^{\circ}$ 406/2009/CE du Parlement Européen et du Conseil du 23 avril 2009 fait état de l'objectif de réduction de $20 \%$ des émissions de GES de l'UE par rapport à 1990. Le Conseil Européen de 2007 s'est également montré favorable à une réduction des émissions de $30 \%$ en cas d'accord international post 2012. Cette décision « partage de l'effort » précise également la réduction fixée pour chaque état membre, dans le secteur qui n'entre pas dans le système européen d'échange de quotas d'émissions (transport, résidentiel et agriculture), pour 2020 par rapport au niveau d'émission de 2005 et notamment une réduction de $14 \%$ pour la France.

La France a souhaité, dans le cadre du Grenelle de l'Environnement (lois Grenelle 1 du 3 août 2009), montrer l'exemple en prévoyant de diviser par quatre ses émissions de gaz carbonique d'ici 2050. Ensuite, la feuille de route de l'Union Européenne pour la réduction des émissions de GES, publiée en mars 2011, fixe un objectif global de réduction de $90 \%$ d'ici 2050 et des jalons par secteur d'activité en 2030 et 2050 . Cette feuille de route fixe notamment pour l'agriculture un objectif de réduction des émissions de 36 à $37 \%$ en 2030 et de 42 à $49 \%$ en 2050 par rapport à 1990. Cette feuille de route, qui n'a pas de valeur juridique contraignante, pourrait cependant servir de référence.

Les réglementations en lien avec l'ammoniac ne sont pas contraignantes à l'heure actuelle pour les élevages de bovins. En termes de qualité de l'air, la France se devait de respecter un plafond d'émission concernant l'ammoniac de 780000 t/an en 2010 alors que les émissions françaises atteignaient 645000 t en 2010 (CITEPA 2010). Ce plafond, défini à l'échelle internationale au travers du Protocole de Göteborg et à l'échelle européenne via la directive " National Emission Ceiling " (Directive 2001/81/NEC) pourrait être prochainement revu à la baisse. Les particules, pour partie liées aux émissions d'ammoniac, sont elles aussi soumises à des plafonds d'émissions, qui régissent les quantités annuelles émises ainsi que les épisodes de pollution ponctuelle (Directive qualité de l'air 2008). Parallèlement, la directive " Industrial Emission Directive » (Directive 2010/75/EU), qui concerne l'application des Meilleures Techniques Disponibles (MTD) ainsi que la déclaration des émissions annuelles en élevage, envisage l'intégration des grands élevages de bovins, au même titre que le sont actuellement les grands élevages de porcs et volailles.

mais aussi de carbone. En bâtiment et au stockage, le type de déjections produites, lisier ou fumier, a une incidence forte sur les émissions de méthane, de protoxyde d'azote et d'ammoniac. Les conditions de production et de stockage des fumiers (en tas sur plateforme ou sous les animaux) agissent notamment sur les niveaux d'émission au travers du degré d'anaérobiose. Des études récentes montrent que les litières accumulées seraient émettrices de $\mathrm{CH}_{4}$ (Edouard et al 2012, Webb et al 2012) en quantité importante comparativement aux lisiers. Ces quantités peuvent représenter de 11 à $18 \%$ du méthane total émis par les animaux et la litière (Hansen et al 2010). Les émissions d'ammoniac des fumiers au stockage dépendent de la teneur en azote soluble et du degré d'aérobiose lié à la densité du tas (Paillat et al 2005). Des stratégies de gestion des fumiers, proposées par Chadwick (2005) pour réduire la volatilisation de l'ammoniac consistent à tasser et bâcher les tas de fumier. Une telle technique nécessiterait toutefois d'être évaluée quant aux émissions de $\mathrm{CH}_{4}$ qui pourraient s'en trouver accrues du fait des conditions d'anaérobioses. Pour les lisiers, la couverture des fosses peut permettre un abattement des émissions d'ammoniac de 60 à $80 \%$ selon le type (toile tendue...). Pour des lisiers de bovins, une « croûte naturelle » formée en surface grâce au taux de matière sèche élevé des lisiers, permet également de réduire les émissions d'ammoniac de $40 \%$. Enfin, la digestion anaérobie des déjections animales sur site constitue un moyen de limiter les émissions de méthane au stockage. En système laitier, l'installation d'une unité de méthanisation peut permettre un abattement des émissions de GES compris entre 5 et $7 \%$ (Dollé et al 2011). Toutefois la mise en œuvre d'un tel dispositif est conditionnée par un certain nombre de paramètres tels que la production constante de déjections, la disponibilité en co-substrats, la valorisation de la chaleur et également l'investissement qu'il représente. Par ailleurs, cette digestion anaérobie implique un risque accru sur les pertes d'ammoniac par volatilisation au stockage et à l'épandage, du fait d'un $\mathrm{pH}$ plus élevé et d'un pool d'azote ammoniacal augmenté. A l'épandage, la volatilisation ammoniacale est principalement liée à la concentration en $\mathrm{NH}_{3}$ du produit, aux conditions climatiques (température, humidité, vent) et au matériel d'épandage (Petersen et al 2011). L'incorporation permettrait de réduire de $30 \%$ les émissions si elle est effectuée dans les 24 heures. Des interactions existent entre les émissions de $\mathrm{NH}_{3}$ et de $\mathrm{N}_{2} \mathrm{O}$ qui néanmoins resteraient plus liées aux conditions pédoclimatiques (types de sol, conditions météorologiques) qu'aux modalités d'apport des engrais organiques (Petersen et al 2011).

Au-delà des flux gazeux vers l'atmosphère, la séquestration du carbone dans les prairies gérées par l'élevage laitier représente un potentiel important d'atténuation des émissions de GES des systèmes d'élevage herbivore (Soussana et al 2010). Ainsi en élevage laitier, la séquestration de carbone sous prairie peut compenser de 6 à $43 \%$ des émissions de GES de l'atelier selon la part de maïs dans la SFP (Dollé et al 2011). L'apport modéré d'azote (engrais, déjections), qui augmente la production de biomasse, accroît la séquestration du carbone alors qu'une carence en azote peut provoquer un déstockage de carbone car les microbes du sol puisent dans les réserves humiques du sol (Fontaine et al 2004, Klumpp et al 2009). La nature du couvert végétal influence également la capacité d'un agro-système à séquestrer du carbone (Loiseau et al 2001). La présence de légumineuses permet une autorégulation en azote et donc le maintien du stock de carbone (Loiseau et al 2001, Soussana et al 2010). Le pâturage permet un meilleur stockage du carbone que la fauche via un apport direct de matière organique par les déjections et une moindre exportation de carbone $\mathrm{du}$ fait de l'herbe résiduelle (Reeder et Schuman 2002, Soussana et Lüscher 2007). Enfin, l'augmentation de la durée de la rotation des prairies temporaires ou la conversion de prairies temporaires en prairies permanentes augmente le stockage de carbone. Par la mise en œuvre de ces différentes pratiques, réduction des apports d'engrais azotés sur des parcelles intensives, conversion des prairies de graminées en mélange de graminées et légumineuses, intensification modérée des prairies pauvres..., le flux de carbone stocké peut ainsi être augmenté de 0,5 à 1,5\% par an.

\section{3 / Elevage laitier et bio- diversité}

Un plan d'action en faveur de la biodiversité en agriculture a été mis en place en 2001 (cf. encadré 3). Les travaux scientifiques publiés sur les effets de l'élevage sur la biodiversité des écosystèmes concluent avec certitude au rôle important de l'élevage laitier (Mc Laughlin et Mineau 1995, Le Roux et al 2008). Ces effets sont cependant complexes et largement méconnus en raison de la très grande diversité des espèces impactées, des écosystèmes à étudier, des échelles spatiales et temporelles 
Encadré 3. Règlementation environnementale en relation avec le maintien de la biodiversité.

Du point de vue des politiques en faveur de la biodiversité, l'élevage est à la fois concerné par la préservation des races animales, et par la préservation des milieux semi-naturels valorisés par l'élevage.

La préservation de la biodiversité est un enjeu international mis en exergue par la conférence de Rio en 1992. Celle-ci a débouché sur la Convention Internationale sur la diversité biologique qui a été ratifiée par la France en 1994. En 1998, la Stratégie Européenne pour la Biodiversité a défini des objectifs généraux pour divers secteurs économiques. Au premier rang desquels figure l'agriculture avec en particulier, la conservation et l'usage durable des agro-écosystèmes. Une évolution majeure des orientations politiques en matière de biodiversité réside dans la prise en compte de la biodiversité " ordinaire ", fortement modulée par les exploitations d'élevage. En 1999, le Conseil Européen d'Helsinki met en place la stratégie d'intégration de l'environnement dans la Politique Agricole Commune (PAC), et celui de Göteborg des 15 et 16 juin 2001 décide de mettre un terme à l'érosion de la biodiversité.

Un plan d'action en faveur de la diversité biologique dans le domaine de l'agriculture qui rappelle les liens étroits existant entre la biodiversité et les pratiques extensives est mis en œuvre en 2001. II recommande de soutenir des pratiques extensives, et les relie explicitement à des instruments communautaires comme les Mesures Agro-Environnementales (MAE) ou les Indemnités Compensatoires de Handicap Naturel (ICHN). En 2004, cet enjeu s'est traduit en France par l'élaboration d'une stratégie nationale pour la biodiversité qui comporte, un plan d'action agriculture articulé en 5 axes prioritaires visant à promouvoir les démarches territoriales et partenariales, généraliser les pratiques agricoles favorables à la biodiversité, protéger et renforcer la diversité des ressources génétiques, mettre en place un observatoire national de l'évolution de la biodiversité en milieu rural en lien avec les pratiques agricoles et renforcer la sensibilisation et les compétences des acteurs. La priorité d'agir pour le maintien de la biodiversité, notamment dans le domaine agricole, a été rappelée en 2009 lors du Grenelle de l'environnement.

à considérer, et des modes d'action de l'animal sur le milieu. En ce qui concerne la biodiversité animale domestique, $82 \%$ des 3,7 millions de vaches laitières recensées en 2012 sont répartis sur deux races, à savoir la Holstein $(65 \%)$ et la Montbéliarde (17\%). Douze races de vaches laitières, qui dénombrent entre 50 et 1500 individus, sont en grandes difficultés. Ces dernières décennies, la standardisation animale en production laitière a affecté de façon inquiétante la biodiversité domestique des races de vaches laitières. Le maintien d'effectifs minimum pour une conservation durable de races à faible effectif nécessite une valorisation économique que seule l'activité d'élevage peut toutefois apporter. L'appréciation de l'impact de l'élevage sur la biodiversité des écosystèmes doit intégrer trois niveaux d'échelle spatiale qui vont de la parcelle au territoire en passant par l'exploitation.

\section{1 / L'échelle de la parcelle agricole}

A l'échelle de la parcelle ou du «patch» de végétation, les pratiques conduites en élevage laitier affectent directement ou indirectement la diversité des communautés végétales et animales. Il en résulte une modification profonde des conditions d'habitat et des ressources alimentaires des organismes (Suominen et Danell 2006). L'intensité du niveau de fertilisation azotée a notamment des effets sur l'abondance des insectes qui est stimulée par la biomasse et le contraste de hauteur d'herbe (McMahon et al 2010).
Le pâturage, par l'intermédiaire de plusieurs processus tels que la déposition d'urines et de fèces, la défoliation, le piétinement..., influence la dissémination des graines et des organismes ainsi que le dérangement ou la destruction d'espèces animales (oiseaux nicheurs des prairies). L'augmentation forte de la pression de pâturage a un effet généralement défavorable sur la richesse en espèces végétales (Isselstein et al 2005) ou animales (Mulder et al 2005). En revanche, des effets inverses sont constatés pour des niveaux plus réduits de pression, en particulier en raison de la création d'une hétérogénéité structurale au sein du couvert végétal prairial. Cette hétérogénéité constitue pour nombre d'espèces floristiques ou faunistiques (insectes, micromammifères et oiseaux), une opportunité de trouver une variété d'habitats, favorables notamment à la diversité de papillons (Sjödin et al 2008). Les niveaux intermédiaires de chargement sont les plus favorables à la biodiversité (Cingolani et al 2005). Le niveau de chargement animal n'est cependant pas la seule modalité à prendre en compte pour comprendre l'impact du pâturage sur la biodiversité à l'échelle de la parcelle. Plantureux (1996) a montré dans une étude sur des prairies lorraines, que la durée de repousse entre deux pâturages avait également un effet positif sur la diversité végétale.

Les effets de l'espèce et de la catégorie animale sont également importants. Les comportements alimentaires sont très contrastés entre espèces (bovins, équins, caprins et ovins) car le mode de préhension de l'herbe sur pied et le comportement de sélection sont différents. Les comportements alimentaires des animaux favorisent ainsi une mosaïque de zones pâturées et de zones de refus servant de refuges aux espèces défavorisées (Muller et al 1998). Contrairement aux vaches, l'apprentissage du pâturage par des jeunes bovins se traduit par une moindre sélectivité pour les repousses (Scimone et al 2007). Néanmoins, les comportements alimentaires sélectifs changent si la hauteur d'herbe diminue; les vaches se reportent alors sur de l'herbe épiée à la différence des brebis par exemple qui poursuivent la consommation de repousses (Dumont et al 2007).

Par ailleurs, dans une synthèse portant sur 197 études conduites à l'échelle mondiale, Díaz et al (2007) ont montré que le pâturage agissait également sur la diversité « fonctionnelle » de la végétation, en favorisant par exemple les plantes de petite taille ou les espèces stolonifères et à architecture en rosette par rapport aux espèces en touffe. Enfin l'abandon total du pâturage, non remplacé par un régime de fauche, se traduit généralement par un embroussaillement qui conduit à un appauvrissement de biodiversité par dominance de quelques espèces (Pykala et al 2005).

\section{2 / L'échelle de l'exploitation agricole}

La dynamique de la richesse spécifique en espèces est très liée à la nature du système de production, avec un effet bénéfique des systèmes de production biologiques (Aude et al 2003), et à l'intensité des pratiques agricoles, une augmentation de l'intensité des pratiques diminuant la diversité végétale (Van Buskirk et Willi 2004). Les décisions de l'éleveur en matière d'assolement, de répartition des parcelles, de mode de récolte (pâture, fauche, type de troupeau) et de gestion des éléments écologiques ont de fait des conséquences importantes sur la biodiversité (Burel et Baudry 1995, Gaujour et al 2012). Par conséquent, l'utilisation et la valorisation par la production laitière de la biodiversité des prairies permanentes favorables à une plus grande souplesse d'exploitation, à l'introduction de légumineuses qui permet de réduire les intrants azotés..., à la présence d'éléments agro-écologiques est un gage de préservation de la biodiversité. Néanmoins, certaines pratiques peuvent avoir des effets négatifs sur la diversité entomologique, tels les anthelminthiques utilisés dans le traitement des animaux domestiques en affectant les insectes associés aux déjections des herbivores dans les prairies (Hutton et Giller 2003). 


\section{3 / L'échelle du paysage agri- cole}

L'intensité des pratiques et des systèmes de production a un impact sur la qualité des éléments du paysage (Le Coeur et al 2002). De nombreuses études établissent des liens entre la complexité du paysage et les systèmes de production (conventionnel, biologique, intégré), ou entre la complexité du paysage et l'intensification de l'agriculture (Herzog et al 2006, Billeter et al 2008). L'abondance et la richesse spécifique en espèces sont fortement influencées par la densité des zones fragmentées dans la matrice paysagère (Jules et Shahani 2003), la taille de ces fragments (figure 3 d'après Krauss et al 2004) et le niveau de connexions entre ces fragments (Van Buskirk et Willi 2004). La mosaïque paysagère, basée, entre autres, sur la proportion des différents éléments agroécologiques, influe également fortement sur la composition des communautés faunistique et floristique (Aviron et al 2005). Cette structure du paysage se mesure par des éléments définis a priori comme les éléments fixes (arbres, haies, bosquets...) dont dépendent souvent les communautés végétales ou animales. Un certain nombre de critères comme l'importance des éléments agro-écologiques et la connectivité des corridors écologiques permet de quantifier et comparer les paysages entre eux. La notion de complexité paysagère est liée à l'importance des éléments agro-écologiques tels les haies, les fossés, les bordures de route, les îlots de végétation non agricole et les bandes enherbées. Considérées sous l'angle de la diversité des habitats refuges (Sullivan et Sullivan 2006, Billeter et al 2008), ces zones contribuent à augmenter la richesse spécifique (Freemark et al 2002) au sein des systèmes d'élevage laitiers.

La connectivité (Deckers et al 2004) a un effet positif sur la diversité végétale spécifique (Lindborg et Eriksson 2004) et la variabilité génétique intra-espèces
(Honnay et al 2007). A cet égard, l'étude des effets de la fragmentation des paysages sur la diversité végétale montre les effets sur différents traits fonctionnels d'espèces végétales comme la dispersion spatiale des graines ou la pérennité des espèces liée à l'état du stock semencier dans ces surfaces (Cousins et al 2003). Au-delà des éléments agroécologiques naturels décrits précédemment, la prairie permanente associée notamment aux systèmes d'élevages laitiers, placée au sein d'une matrice paysagère contribue également au maintien d'une richesse spécifique élevée (Eriksson et al 2002, Cousins et al 2003).

\section{4 / Quantification des impacts environnementaux des sys- tèmes d'élevage laitier}

En réponse aux enjeux décrits précédemment, les systèmes laitiers nationaux ont fait l'objet d'une évaluation des impacts environnementaux relatifs à la préservation de la qualité de l'eau, de la qualité de l'air et de la biodiversité.

\section{1 / Méthodes mises en œuvre pour l'évaluation environnemen- tale des systèmes de production}

Une analyse environnementale multicritère (Manneville et al non publié) basée sur l'Analyse du Cycle de Vie (ACV) a été conduite sur un échantillon de 206 exploitations laitières spécialisées de la base de données des réseaux d'élevage pour l'année 2010. Le périmètre d'évaluation concerne l'ensemble des impacts sur la partie du cycle de vie du produit s'arrêtant au portail de la ferme. Cette évaluation intègre les impacts directs liés au processus de production au niveau de l'atelier laitier, mais également les impacts indirects inhérents à la fabrication des intrants et à leur transport (aliments, engrais, carburants...). L'ensemble des impacts environnementaux sur l'eau, l'air et la biodiversité ont été considérés. L'impact potentiel sur la qualité de l'eau est évalué au travers de l'indicateur potentiel d'eutrophisation. Cet indicateur d'eutrophisation, exprimé en $\mathrm{kg}$ éq. $\mathrm{PO}_{4}$, incombe à l'azote lixivié et au phosphore ruisselé. L'impact de l'élevage laitier sur la qualité de l'air est apprécié au travers des émissions gazeuses azotées et carbonées qui contribuent d'une part, au phénomène de changement climatique et d'autre part, à la volatilisation de l'ammoniac qui participe au phénomène d'acidification atmosphérique. L'évaluation de l'impact sur le changement climatique, repose sur la méthodologie GES'TIM (Gac et al 2010) qui intègre les émissions de méthane, de protoxyde d'azote et de gaz carbonique associées à la combustion des énergies fossiles. Basé sur le pouvoir de réchauffement global des différents gaz $(25 \mathrm{~kg} \mathrm{CO} / \mathrm{kg}$ de $\mathrm{CH}_{4}$ et $298 \mathrm{~kg} \mathrm{CO}_{2} / \mathrm{kg}$ de $\mathrm{N}_{2} \mathrm{O}$ ), 1 'impact sur le changement climatique ou empreinte carbone est exprimé en kg éq. $\mathrm{CO}_{2}$. Le potentiel d'acidification atmosphérique, en lien avec les émissions d'ammoniac, s'exprime en kg éq. $\mathrm{SO}_{2}$. La contribution au maintien de la biodiversité est quant à elle mesurée par dénombrement des éléments agro-écologiques (prairies, haies, bosquets, bois, cours d'eau...) traduits en Surface Equivalente Topographique (SET) selon les coefficients retenus dans les Bonnes Conditions Agricoles et Environnementales (BCAE) VII et la Prime à l'Herbe Agri-Environnementale (PHAE) 2. Les résultats sont exprimés en $\mathrm{m}^{2}$ éq. d'éléments agro-écologiques.

L'évaluation des impacts environnementaux pose ensuite la question de l'Unité Fonctionnelle (UF), c'est-à-dire du mode d'expression des résultats. Dans une approche produit/filière, l'UF est généralement la quantité de produit, exprimée en poids ou unité de volume (kg ou litre de lait), afin d'appréhender les impacts liés à la production du bien sur son cycle de vie. Il peut également

Figure 3. Effet de la taille des fragments d'habitats sur les espèces végétales spécialistes et généralistes (Krauss et al 2004).
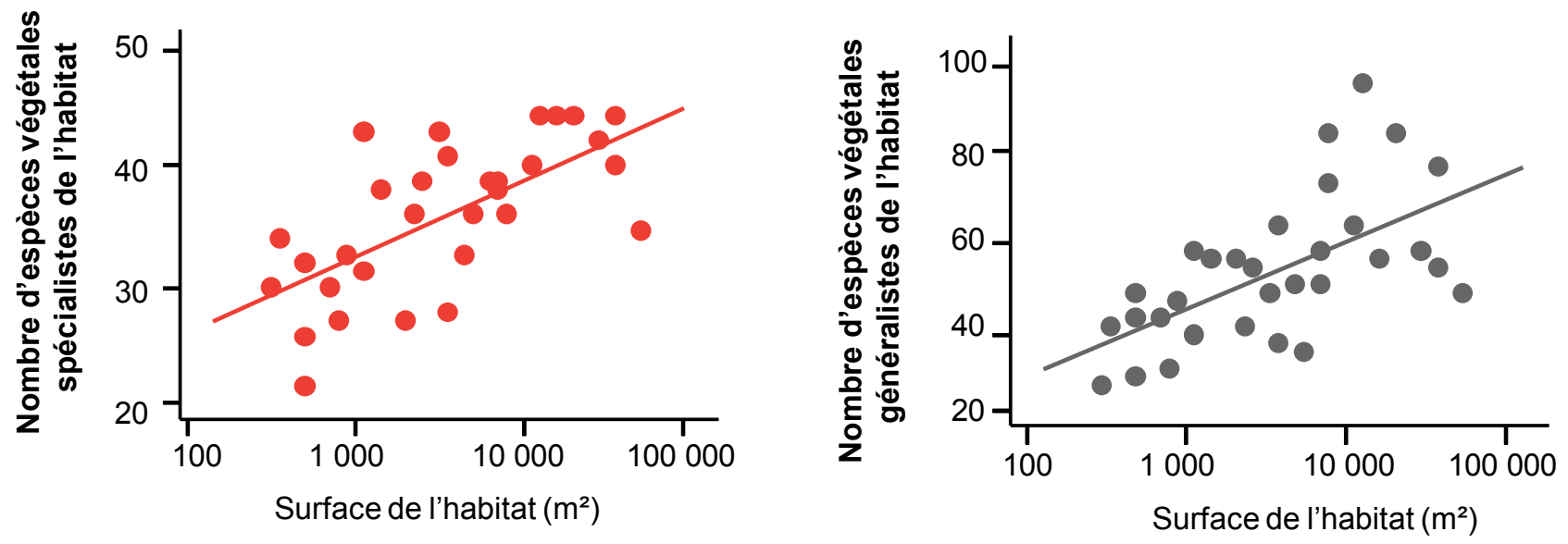
s'agir d'une unité relative à un critère de qualité ou à une fonctionnalité recherchée ( $\mathrm{kg}$ de protéines, kcal d'énergie brute, MJ d'énergie potentielle...). Ces modes d'expression des impacts, qui intègrent la notion de rendement, sont toutefois insuffisants dans la mesure où ils ne traduisent pas la pression réelle exercée sur le milieu par l'activité en question. Les impacts peuvent ainsi être ramenés à l'ha lorsque les indicateurs sont en lien direct avec la surface mobilisée (eutrophisation, biodiversité...). Dans le cadre de nos travaux, les UF retenues sont le litre de lait et la SAU de l'atelier mobilisée pour la production laitière.

Les impacts environnementaux relatifs à l'atelier laitier concernent l'ensemble des produits commercialisés par cet atelier, à savoir le lait mais également la viande issue des réformes laitières. La répartition des impacts entre les différents produits nécessite d'avoir recours à un moyen d'allocation des impacts (Dollé et al 2011). Plusieurs modes d'allocation entre le lait et la viande sont identifiés (biophysique, protéique, économique...). Le mode d'allocation biophysique, retenu dans le cadre de l'évaluation des impacts des systèmes laitiers nationaux, est ici basé sur le fonctionnement du système d'exploitation. Le principe de ce mode d'allocation est de considérer que les génisses laitières, qui seront de futures vaches de réforme, contribuent à la production de viande et ainsi d'associer les impacts environnementaux de cette catégorie animale à la viande valorisée en réforme laitière. Parallèlement les impacts associés aux vaches laitières sont affectés à la production de lait. Pour rendre son application aisée, ce mode d'allocation repose sur l'énergie consommée par les deux catégories animales pour produire respectivement la viande et le lait. Nous considérons ainsi que l'énergie consommée par les génisses est destinée à la production de viande et celle des vaches en lactation à la production de lait, à l'exception de l'énergie de la gestation associée à la croissance du veau. L'application de ce mode de partition de l'énergie totale consommée par un atelier laitier, met en évidence que $74 \%$ de l'énergie concerne les vaches en production, $3 \%$ les veaux et $23 \%$ les génisses de renouvellement (Dollé et al 2013). Appliqués aux impacts totaux de l'atelier, ces ratios permettent l'allocation des impacts aux deux produits lait et viande.

\section{2 / Impacts environnementaux moyens et variabilité}

Sur les 206 exploitations de l'échantillon, l'empreinte carbone brute est de $1,0 \mathrm{~kg} \mathrm{CO}_{2} /$ litre de lait (tableau 3). Cette empreinte carbone est conforme aux valeurs rencontrées dans la bibliographie (Vellinga et al 2011), mais inférieure aux résultats obtenus sur un échantillon de 153 fermes laitières françaises par Dollé et al 2011. Cette différence, en partie liée à l'échantillon d'exploitations, est principalement due au mode d'allocation des impacts. Le mode d'allocation protéique appliqué par Dollé et al 2011, contribuait en effet à affecter une part plus importante des impacts au lait (85\%) comparativement au mode d'allocation biophysique (74\%). La prise en considération du stockage de carbone par les prairies et les haies, qui permet une compensation de $30 \%$ des émissions, conduit à une empreinte nette de $0,7 \mathrm{~kg}$ $\mathrm{CO}_{2}$ /litre de lait. Les indicateurs eutrophisation et acidification, respectivement de $0,003 \mathrm{~kg}$ éq. $\mathrm{PO}_{4} /$ litre de lait et $0,009 \mathrm{~kg}$ éq. $\mathrm{SO}_{2} /$ litre de lait, sont conformes aux données de la bibliographie (Cederberg et Flysjo 2004, BassetMens et al 2009). Ils sont cependant inférieurs à la valeur déterminée par

Tableau 3. Performances techniques et environnementales de 206 élevages laitiers représentatifs des systèmes français de plaine et de montagne.

\begin{tabular}{|c|c|c|c|c|}
\hline & & Echantillon & Plaine & Montagne \\
\hline \multicolumn{2}{|c|}{ Nombre d'exploitations } & 206 & 128 & 78 \\
\hline \multirow{8}{*}{ 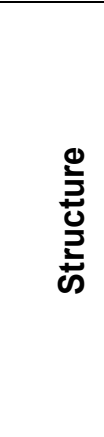 } & Nombre de vaches laitières & 56 & 62 & 46 \\
\hline & SAU (ha) & 89 & 94 & 74 \\
\hline & SFP (ha) & 72 & 71 & 67 \\
\hline & Lait produit (L) & 359702 & 403228 & 289892 \\
\hline & Lait standard produit par vache (L/VL) & 6434 & 6675 & 6200 \\
\hline & Surface Toujours en Herbe ( $\%$ de la SAU) & 40 & 29 & 55 \\
\hline & Surface en maïs (\% de la SFP) & 16 & 22 & 9 \\
\hline & Chargement (UGB/ha SFP) & 1,3 & 1,4 & 1,1 \\
\hline \multirow{5}{*}{ 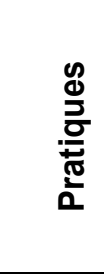 } & Quantité de concentrés (g/L lait) & 216 & 191 & 261 \\
\hline & Rejets azotés (kg N/UGB) & 95 & 92 & 100 \\
\hline & Quantité d'azote minéral (kg N/ha SAU) & 44 & 52 & 33 \\
\hline & Bilan azoté (hors fixation, $\mathrm{kg} \mathrm{N} / \mathrm{ha} \mathrm{SAU}$ ) & 49 & 54 & 43 \\
\hline & $\mathrm{N}$ lessivé (kg N/ha SAU) & 35 & 45 & 21 \\
\hline \multirow{7}{*}{ 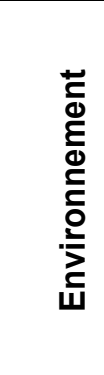 } & Empreinte carbone brute $\left(\mathrm{kg}\right.$ éq $\left.\mathrm{CO}_{2} / \mathrm{L}\right)$ & 1,0 & 0,9 & 1,0 \\
\hline & Empreinte carbone nette ( $\mathrm{kg}$ éq $\mathrm{CO}_{2} / \mathrm{L}$ ) & 0,7 & 0,7 & 0,6 \\
\hline & Acidification ( $\mathrm{kg}$ éq $\mathrm{SO}_{2} / \mathrm{L}$ ) & 0,009 & 0,008 & 0,009 \\
\hline & Eutrophisation ( $\mathrm{kg}$ éq $\mathrm{PO}_{4} / \mathrm{L}$ ) & 0,003 & 0,003 & 0,003 \\
\hline & Eutrophisation (kg éq $\mathrm{PO}_{4} / \mathrm{ha} \mathrm{SAU}$ ) & 26 & 31 & 19 \\
\hline & Biodiversité $\left(\mathrm{m}^{2} / \mathrm{L}\right)$ & 2,4 & 2,1 & 3,3 \\
\hline & Biodiversité (ha éq/ha SAU) & 1,2 & 1,04 & 1,44 \\
\hline
\end{tabular}


Van Der Werf et al (2009) pour l'eutrophisation, $0,006 \mathrm{~kg}$ éq. $\mathrm{PO}_{4} /$ litre de lait et supérieurs pour l'acidification, $0,007 \mathrm{~kg}$ éq. $\mathrm{SO}_{2} /$ litre de lait. La contribution moyenne à la biodiversité est de 2,4 $\mathrm{m}^{2}$ d'éléments agro-écologiques/ litre de lait. Peu de références sont aujourd'hui disponibles pour ce nouvel indicateur, cependant les valeurs observées sont proches de la valeur moyenne de $2,1 \mathrm{~m}^{2} /$ litre de lait déterminée en 2010 par l'Institut de l'Elevage sur un échantillon de 249 fermes laitières françaises.

Les écarts observés entre les exploitations de plaine et de montagne sont non significatifs sur les indicateurs de changement climatique, d'acidification et d'eutrophisation, exprimés par litre de lait (tableau 3), malgré des différences notables en matière de consommation de concentrés et d'engrais minéraux. Les résultats sont à l'avantage des exploitations de montagne sur le stockage de carbone et la contribution à la biodiversité en raison d'une part plus élevée de prairies dans le système. L'expression des impacts par $\mathrm{kg}$ de lait cache toutefois des disparités en matière de pression exercée sur le milieu entre les exploitations de plaine et de montagne. L'indicateur d'eutrophisation, ramené à l'ha de SAU, est ainsi plus faible pour les exploitations de montagne comparativement aux exploitations de plaine compte tenu des plus faibles apports d'engrais de synthèse (tableau 3 ) et de la part d'herbe dans le système $(55 \%$ en zone de montagne vs $29 \%$ en zone de plaine). De même, la densité d'éléments agroécologiques à l'hectare est plus forte dans les exploitations de montagne du fait de la part d'herbe dans le système en lien avec la mosaïque paysagère plus développée.

Une analyse plus approfondie distinguant les systèmes de production selon la part d'herbe et de maïs dans la ration (figure 4) renforce cet effet positif des prairies sur la limitation des risques d'eutrophisation. Associé à une pression d'azote minéral moindre et à une lixiviation plus faible de l'azote sur prairies comparativement au maïs, les systèmes herbagers possèdent un impact eutrophisation plus faible quelle que soit la zone. En revanche, contrairement à l'eutrophisation, aucune variabilité de l'empreinte carbone brute n'est constatée selon la part d'herbe dans le système de production. La situation est toutefois différente dès lors que l'on intègre le stockage de carbone par les prairies.

Alors que les différences entre zones de production (plaine $v s$ montagne) sont faibles, une forte variabilité intra zone de production est mise en évidence. Ces différences entre exploitations d'une
Figure 4. Impact eutrophisation de cinq systèmes laitiers.

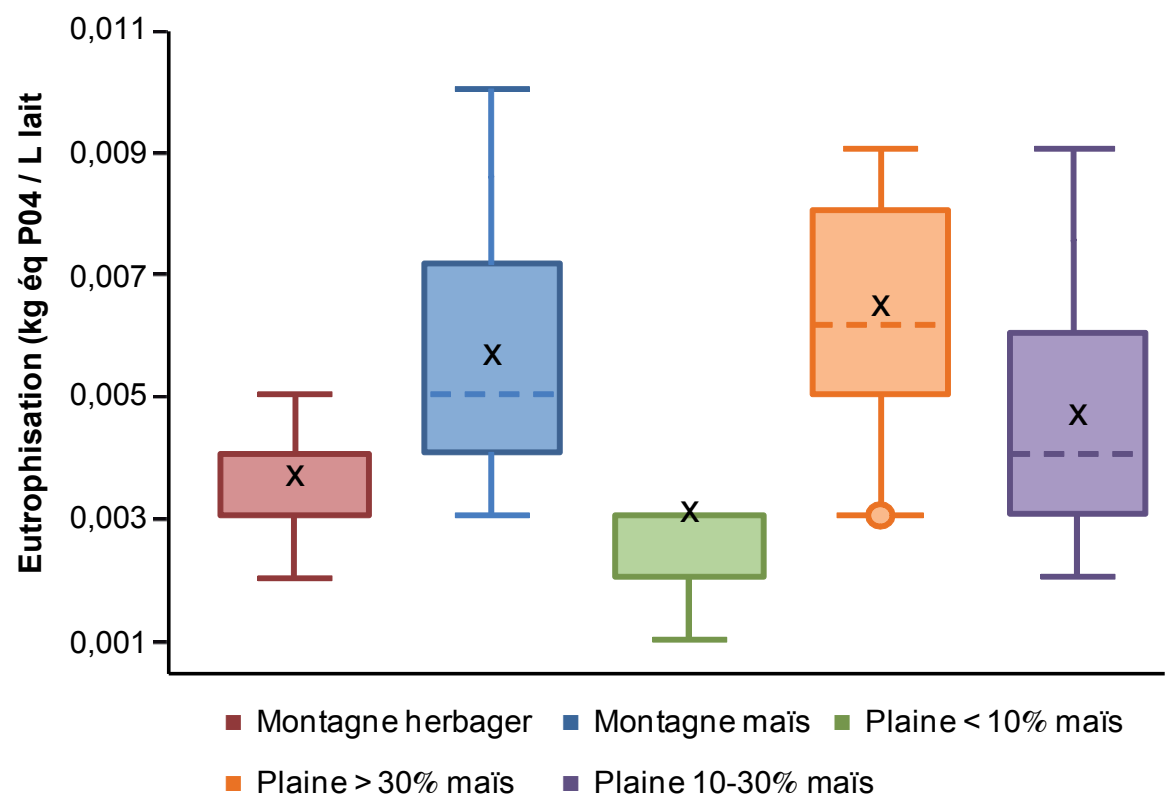

même zone, comprises entre 20 et $30 \%$, sont observées pour l'ensemble des indicateurs environnementaux. Il ressort donc que les variations d'empreinte environnementale sont davantage liées à la gestion du troupeau et aux pratiques qu'au système de production et à sa zone d'appartenance.

\section{3 / Incidences des pratiques et de la structure d'exploitation sur les résultats environnementaux}

Sur ce même échantillon d'exploitations, une Analyse en Composantes Principales (ACP) et une Classification Ascendante Hiérarchique (CAH) ont été réalisées afin d'identifier les exploitations les plus efficientes d'un point de vue environnemental et le lien avec les pratiques. Réalisée sur les exploitations de plaine et de montagne afin de piéger l'effet de la structure et l'effet des pratiques, cette analyse a permis une analyse comparée de types d'exploitations se distinguant par les résultats techniques et environnementaux.

Qu'il s'agisse des exploitations de plaine ou de montagne, les exploitations efficientes d'un point de vue environnemental et optimisées sur le plan technique possèdent de faibles empreintes carbone, eutrophisation et acidification (tableau 4). Les composantes structurelles des exploitations optimisées et non optimisées (part de maïs...) étant comparables pour les exploitations des deux zones géographiques, les résultats environnementaux distincts sont liés à trois facteurs principaux :

Le niveau de dépendance aux intrants (concentrés, engrais minéraux...). Le recours aux intrants influe sur les impacts environnementaux directs et indirects du processus de production (Henrikson et al 2011). Pour les exploitations de plaine et de montagne, les systèmes efficients d'un point de vue environnemental se caractérisent par un faible recours aux intrants (concentrés, fertilisants azotés et phosphatés) comparativement aux autres systèmes moins autonomes. Ce manque d'autonomie et le défaut d'ajustement des apports aux besoins se traduisent par une dépendance énergétique forte, des bilans azotés très supérieurs..., le tout étant à l'origine d'impacts environnementaux élevés. de renouvellement, les vaches présentant des problèmes sanitaires (boiteries, mammites...) influent sur le nombre d'animaux " improductifs », le chargement animal et les performances environnementales. A même niveau de productivité par vache, un niveau de chargement distinct traduit une gestion différenciée des effectifs animaux. ne et de montagne les moins optimisées, présentant une part d'animaux « improductifs » plus élevée, possèdent un chargement animal et des impacts environnementaux supérieurs. Le temps passé carbonées et azotées supérieures, influe également sur les résultats environnementaux. Non discriminant pour les systèmes de montagne où la durée de stabulation est voisine de 190 jours, le temps de présence en bâtiment influe sur les résultats environnementaux des systèmes de plaine. Ainsi, les systèmes qui assurent une meilleure valorisation de l'herbe (temps au pâturage supérieur) possèdent de plus faibles impacts environnementaux du fait de la réduction des émissions sur le segment bâtimentstockage.
La gestion du troupeau. Les effectifs C'est ainsi que les exploitations de plaien bâtiment, associé à des émissions 
Tableau 4. Performances technico-économiques et environnementales de deux types d'exploitations selon leur niveau d'optimisation technique en zones de plaine et de montagne.

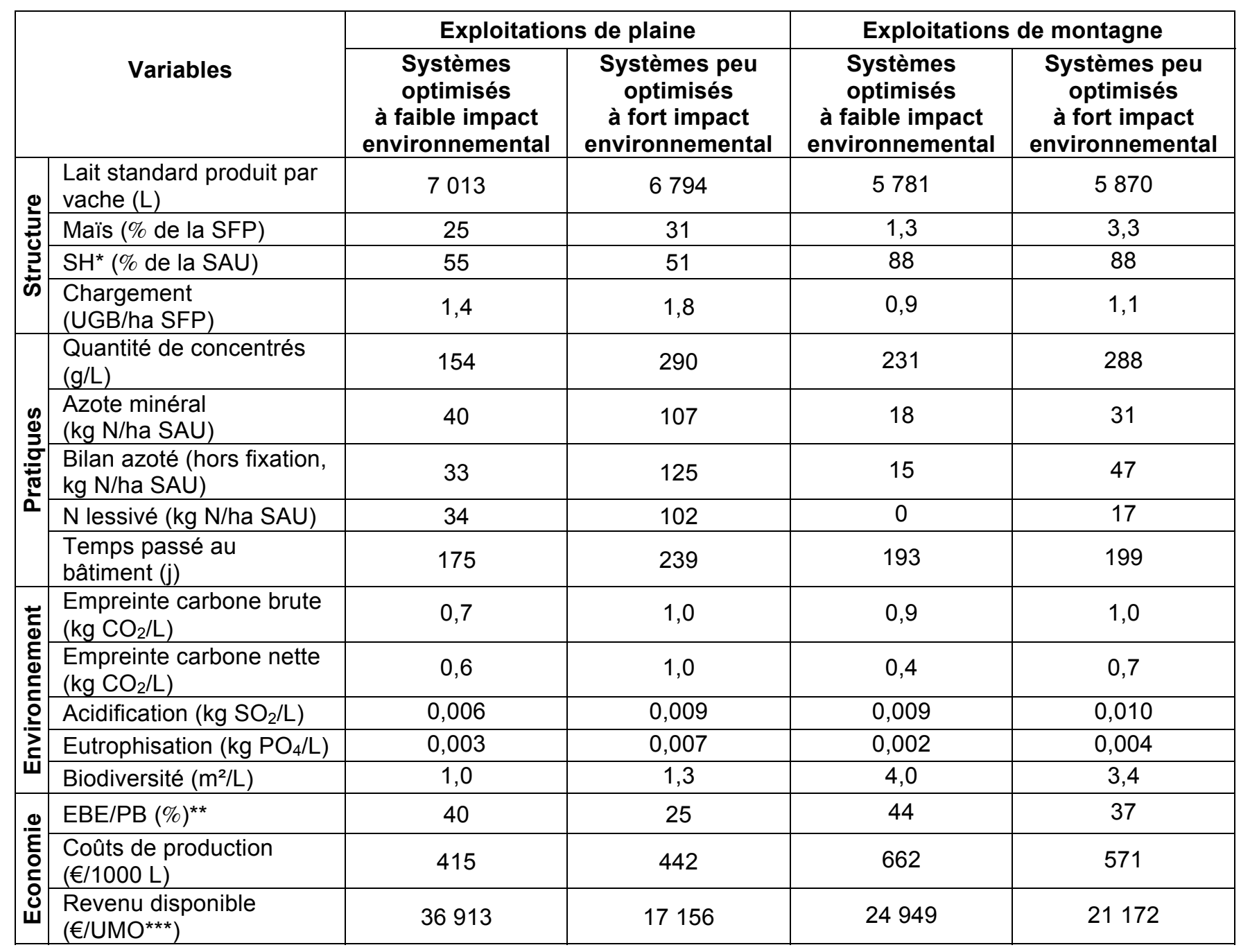

* SH : Surface en Herbe.

** EBE : Excédent Brut d'Exploitation; PB : Produit brut.

*** UMO : Unité de Main-d'œuvre.

La productivité laitière. A même niveau d'intrants, même effectifs, etc. une productivité par vache supérieure est un gage de réduction de l'empreinte carbone au litre de lait (Schils et al 2005, Vellinga et al 2011). Les systèmes de plaine, où la productivité par vache est légèrement différente, permettent de mettre en évidence l'effet du niveau de productivité entre les systèmes optimisés et non optimisés. Cette tendance est toutefois à relativiser dans la mesure où certains gains de productivité, associés à un recours plus important aux intrants, un temps de présence des animaux en bâtiment plus long, des risques sanitaires accrus, une part plus faible de prairies..., se traduisent dans de nombreuses situations par des impacts environnementaux supérieurs ramenés au litre de lait; et dans tous les cas par une pression plus forte sur le milieu.

\section{4 / Liens entre efficiences éco- nomique et environnementale}

La confrontation des données techniques, qui permettent de caractériser le degré d'optimisation des exploitations (intrants, dépendance énergétique, productivité laitière...), aux résultats économiques (coût de production, revenu disponible) et environnementaux met en évidence le lien entre efficacité technique, économique et environnementale (tableau 4). Les exploitations les plus performantes sur le plan technique, qui connaissent de faibles impacts environnementaux, ont les meilleurs résultats économiques. A l'inverse, les exploitations non optimisées d'un point de vue environnemental ont recours de façon importante aux intrants (engrais, aliments, énergies directes) et possèdent des coûts de production plus conséquents. Conformément aux observations faites par Jan et al (2011), les systèmes optimisés (fertilisation, ration alimentaire) valorisant les surfaces en herbe présentent ainsi de bons résultats économiques et environnementaux renforçant le lien entre performances technico-économiques et environnementales. De même, le niveau de technicité dans la gestion et la valorisation agronomique des déjections animales, et donc leur recyclage, en lien avec le type d'infrastructures et d'équipements, est également une garantie de réussite technique, économique et environnementale. Enfin, les moyens développés pour augmenter l'autonomie alimentaire des exploitations en jouant sur les concentrés et/ou les fourrages, représentent une alternative pour améliorer revenu et bilans environnementaux. Par ailleurs, toutes les bonnes pratiques de gestion du troupeau sur le plan sanitaire et de la reproduction, et qui réduisent le nombre d'animaux « improductifs », sont ainsi un gage de réussite économique et environnementale. C'est ainsi que les éleveurs qui ont diminué les achats d'engrais et amélioré la productivité par vache entre 
1990 et 2010 ont d'une part, réduit de 15 à $25 \%$ leur empreinte carbone et de 10 à $66 \%$ l'impact eutrophisation et, d'autre part, abaissé les coûts de production (Dollé et al 2013). C'est pourquoi, préalablement à la mise en œuvre de nouvelles techniques complexes et parfois onéreuses, nombre de solutions techniques sont d'ores et déjà disponibles et applicables en élevage à moindre coût, voire avec un intérêt économique certain pour l'éleveur.

\section{Conclusion}

Dans un contexte d'évolution des enjeux environnementaux élargis à de nouvelles thématiques (émissions gazeuses, biodiversité...), l'élevage laitier présente la particularité d'être un émetteur de polluants (GES, ammoniac, nitrates...) tout en occupant un rôle central dans la préservation de l'environnement au travers de la séquestration du carbone et de la contribution au maintien de la biodiversité. L'évaluation environnementale multicritère passe par une approche intégrée à l'échelle de l'exploitation et non uniquement sectorielle afin d'éviter tout transfert d'impact d'un poste à l'autre. Elle permet de pointer les impacts environnementaux et les contributions positives des systèmes de production tout en mettant en lumière également les risques de transfert d'impact d'un compartiment à l'autre. Une telle approche multicritère à l'échelle de l'atelier laitier, mais également à l'échelle de l'exploitation permettra d'une part, de valoriser les complémentarités et les synergies entre productions et, d'autre part, la construction de plans d'action environnementaux cohérents. Il convient toutefois de souligner que ces approches multicritères sont encore récentes et l'incidence que peuvent avoir le périmètre d'analyse, les facteurs d'émission retenus, les méthodes d'allocation des impacts, les unités employées etc, sur le résultat final. D'importants travaux méthodologiques doivent pour cela être conduits au niveau national et international afin de mutualiser et homogénéiser les approches. C'est notamment le cas des travaux en cours qui visent à agréger l'ensemble des composantes des pratiques agricoles et de l'organisation spatiale des éléments agro-écologiques dans le but de constituer un indicateur d'état de la biodiversité (Chanséaume et al 2013).

Sur la base des méthodes existantes, l'évaluation conduite sur les systèmes d'élevage rencontrés en France, ne met en évidence aucune différence notable entre les systèmes de plaine et de montagne, les systèmes intensifs et extensifs, etc. Toutefois, le recours aux prairies a une incidence positive marquée sur le potentiel d'eutrophisation, le stockage de carbone et la contribution à la biodiversité. Au-delà de cet effet « prairies », une très forte variabilité est observée entre exploitations d'un même système, variabilité qui donne à chaque exploitation les moyens d'optimiser ses pratiques en vue de réduire les impacts environnementaux, sans remettre en cause ses performances économiques. Ces pratiques, axées notamment sur le recyclage du carbone et de l'azote, concernent la gestion des déjections animales, la valorisation du pâturage (réduction des émissions en bâtiment et de la consommation énergétique...), la réduction du recours aux intrants (concentrés, fertilisants...) et aussi la gestion des effectifs (aspects sanitaires, reproduction, âge au premier vêlage...) et la réduction de la dépendance énergétique. Le lien entre efficience technico-économique et environnementale des exploitations illustre les opportunités offertes aux éleveurs pour réduire leurs impacts environnementaux sans incidence négative sur la durabilité économique. Audelà de l'optimisation technico-économiques et environnementales accessible à court terme, d'autres leviers ou techniques innovantes devront être actionnés afin de réduire plus avant les impacts environnementaux. Ces leviers, qui pourront générer des frais de fonctionnement ou d'investissement (couverture des ouvrages de stockage, équipements pour la gestion des déjections, unités de méthanisation...) ne devront toutefois pas remettre en cause la pérennité des systèmes de production. Pour ce faire, ils doivent intégrer les dimensions économiques et sociales.

\section{Références}

Aarts H.F.M., Habekotte B., Van Keulen H., 2000. Nitrogen $(\mathrm{N})$ management in the 'De Marke' dairy farming system, Nutr. Cycl. Agroecosyst. 56, 231-240

Aude E., Tybirk K., Bruus Pedersen M., 2003. Vegetation diversity of conventional and organic hedgerows in Denmark. Agric., Ecosys. Environ., 99, 135-147.

Aufrère J., Théodoridou K., Baumont R., 2012. Valeur alimentaire pour les ruminants des légumineuses contenant des tannins condensés en milieux tempérés. INRA Prod. Anim., 25, 29-44.

Aviron S., Burel F., Baudry J., Schermann N., 2005. Carabid assemblages in agricultural landscapes: impacts of habitat features, landscape context at different spatial scales and farming intensity. Agric. Ecosys. Environ., 108, 205-217.

Barré C., 2001. Devenir de l'azote des pissats de vache émis sur une prairie pâturée. Thèse ENSA Rennes, 140p.

Basset-Mens C., Ledgard S., Boyes M., 2009. Eco-efficiency of intensification scenarios for milk production in New Zealand. Ecol. Econ. 68, 1615-1625.

Billeter R., Liira J., Bailey D., Bugter R., Arens P., Augenstein I., Aviron S., Baudry J.,
Bukacek R., Burel F., Cerny M., De Blust G., De Cock R., Diekotter T., Dietz H., Dirksen J., Dormann C., Durka W., Frenzel M., Hamersky R., Hendrickx F., Herzog F., Klotz S., Koolstra B., Lausch A., Le Coeur D. Maelfait J.P., Opdam P., Roubalova M., Schermann A., Schermann N., Schmidt T., Schweiger O., Smulders M.J.M., Speelmans M., Simova P., Verboom J., van Wingerden W.K.R.E., Zobel M. 2008. Indicators for biodiversity in agricultural landscapes: a pan-European study. J. Appl. Ecol., 45, 141-150.

Bolan N.S., Surinder S., Luo J., Rita B., Jagrati S., 2004. Gaseous emissions of nitrogen from grazed pastures: Processes, measurements and modelling, environmental implications, and mitigation. Adv. Agron., 84, 37-120.

Burel F., Baudry J., 1995. Species biodiversity in changing agricultural landscapes: A case study in the Pays d'Auge, France. Agric. Ecosys. Environ., 55, 193-200.

Calsamiglia S., Ferret A., Reynolds C.K., Kristensen N.B., van Vuuren A.M., 2010. Strategies for optimizing nitrogen use by ruminants. Animal, 4, 1184-1196.

Carpentier B., Cabon G., 2011. Le maïs fourrage : élaboration du rendement et de la qualité, récolte et conservation. Fourrages, 205, 11-23.
Cederberg C., Flysjö A., 2004. Life Cycle Inventory of 23 Dairy Farms in South-Western Sweden, Göteborg : Sik.http://www.sik.se/ archive/pdf-filerkatalog/SR 728(1).pdf

Chadwick D.R., 2005. Emissions of ammonia, nitrous oxide and methane from cattle manure heaps: effect of compaction and covering. Atmosph. Environ., 39, 787-799.

Chanséaume A., Blanchet R, Lagache T. Manneville V., Michel N., Amiaud B., 2013. Mixed effects of agricultural practices and agro-ecological structures on grassland plant diversity. The role of grasslands in a green future. Proc. 17 ${ }^{\text {th }}$ Symp. Europ. Grassland Fed., 23-26 Juin, Akureyri, Iceland.

Chevassus-au-Louis B., Salles J.M., Pujol J.L., 2009. Approche économique de la biodiversité et des services liés aux écosystèmes. Centre d'analyse stratégique, Rapports et documents, 376p

Chevassus-au-Louis B., Femenias A., Andral B., Bouvier A., 2012. Bilan des connaissances scientifiques sur les causes de prolifération des macroalgues vertes. Application à la situation de la Bretagne et proposition. Rapport CGAAER N ${ }^{\circ} 11128,147 \mathrm{p}$.

Cingolani A.M., Posse G., Collantes M.B. 2005. Plant functional traits, herbivore 
selectivity and response to sheep grazing in Patagonian steppe grasslands. J. Appl. Ecol., 42, 50-59.

CITEPA, 2010. http://www.citepa.org/fr/pollution-et-climat/polluants/aep-item/ammoniac CN.
$180 \mathrm{p}$.

CNIEL, 2012. L'économie laitière en chiffres,

Cousins S., Lavorel S., Davies I.D., 2003. Modelling the effects of landscape pattern and grazing regimes on the persistence of plant species with high conservation value in grasslands in south-eastern Sweden. Landscape Ecol., 18, 315-332.

Decau M.L., Delaby L., Roche B., 1997. AzoPât : une description quantifiée des flux annuels d'azote en prairie pâturée par les vaches laitières. II les flux du système solplante. Fourrages, 151, 313-330.

Decau M.L., Simon J.C., Jacquet A., 2003. Fate of urine nitrogen in three soils throughout a grazing season. J. Environ. Qual., 32, 14051413.

Decau M.L., Simon J.C., Jacquet A., 2004. Nitrate leaching under grassland as affected by mineral nitrogen fertilization and cattle urine. J. Environ. Qual., 33, 637-644.

Deckers B., Hermy M., Muys B., 2004. Factors affecting plant species composition of hedgerows: relative importance and hierarchy. Acta Oecol., 26, 23-37.

Delaby L., Peyraud J.L., Vérité R., 1995. Influence du niveau de production laitière et du système d'alimentation sur les rejets azotés du troupeau. Renc. Rech. Rum., 2, 349-354.

Delaby L., Lucbert J., 1999. Estimation des flux d'azote, de phosphore et de potassium associés aux vaches laitières et à leur système fourrager. CORPEN, Paris, France, 18p.

Demarquilly C., Chenost M., Giger S., 1995. Pertes fécales et digestibilité des aliments et des rations. In Nutrition des ruminants domestiques, Ed Jarrige et coll., INRA, Paris, France, 601-647.

Devun J., Brunschwig P., Guinot C., 2012. Alimentation des bovins : rations moyennes et autonomie alimentaire, Compte rendu résultats 001239 005, Institut de l'Elevage, Paris, France, 46p.

Diaz S., Lavorel S., McIntyre S., Falczuk V., Casanoves F., Milchunas D.G., Skarpe C., Rusch G., Sternberg M., Noy-Meir I., Landsberg J., Zhang W., Clark H., Campbell B.D., 2007. Plant trait responses to grazing - A global synthesis. Global Change Biol., 13, 313341 .

DIRECTIVE 2001/81/EC of the European Parliament and of the Council of 23 october 2001 on national emission ceilings for certain atmospheric pollutants.

DIRECTIVE 2010/75/EU of the European Parliament and of the Council of 24 november 2010 on industrial emissions (integrated pollution prevention and control).

Dollé J.B., Agabriel J., Peyraud J.L., Faverdin P., Manneville V., Raison C., Gac A., Le Gall A., 2011. Les gaz à effet de serre en élevage bovin : évaluation et leviers d'action. In: Gaz à effet de serre en élevage bovin : le méthane. Doreau M., Baumont R., Perez J.M. (Eds). Dossier, INRA Prod. Anim., 24, 415-432.

Dollé J.B., Manneville V., Gac A, Charpiot A., 2011. Emissions de gaz à effet de serre et consommations d'énergie des viandes bovines et ovines françaises : revue bibliographique et évaluations sur l'amont agricole. Institut de l'Elevage, Paris, France, 59p.

Dollé J.B., Faverdin P., Agabriel J., Sauvant D., 2013. Contribution de l'élevage bovin aux émissions de GES et au stockage de carbone selon les systèmes de production. Actes des Journées AFPF, 26 et 27 Mars, Paris, France, $16 \mathrm{p}$.

Doreau M., Dollé J.B., 2011. Strategies for reducing greenhouse gas emissions in dairy production. A European perspective. Proc. Eastern Nutr. Conf. 12-13 avril 2011, Montréal, Canada, 57-77.

Dulphy J.P., Grenet N., 2001. Estimation des flux d'azote, de phosphore et de potassium associés aux bovins allaitants et aux bovins en croissance ou à l'engrais, issus des troupeaux allaitants et laitiers et à leur système fourrager. CORPEN, Paris, France, 34p.

Dumont B., Farrugia A., Garel J.P., 2007. Pâturage et biodiversité des prairies permanentes. Renc. Rech. Rum., 17-24.

Edouard N. Charpiot A., Hassouna M., Faverdin P., Robin P., Dollé J.B., 2012. Ammonia and greenhouse gases emissions from dairy cattle buildings: slurry $v s$. farm yard manure management systems. Int. Symp. Emissions of Gas and dust from Livestock. EMILI - Saint-Malo, France,

Eriksson O., Cousins S.A.O., Bruun H.H., 2002. Land use history and fragmentation of traditionally managed grasslands in Scandinavia. J. Veget. Sci., 13, 743-748.

Fontaine S., Bardoux G., Abbadie L., Mariotti A., 2004. Carbon imput to soil may decrease soil carbon content. Ecol. Letters, 7, 314-320.

Freemark K., Bert D., Villard M.-A., 2002. Patch-, landscape-, and regional-scale effects on biota. In: Applying Landscape Ecology in Biological Conservation, Gutzwiller K.J. (Ed), Springer-Verlag, New York, 58-83.

Gac, A., Béline F., Bioteau T., Maguet K., 2007. A French inventory of gaseous emissions $\left(\mathrm{CH}_{4}, \mathrm{~N}_{2} \mathrm{O}, \mathrm{NH}_{3}\right)$ from livestock manure management using a mass-flow approach. Livest. Sci., 112, 252-260.

Gac A., Deltour L., Cariolle M., Dollé J.B., Espagnol S., Flénet F., Guingand N., Lagadec S., Le Gall A., Lellahi A., Malaval C., Ponchant P., Tailleur A., 2010. GES'TIM, Guide méthodologique pour l'estimation des impacts des activités agricoles sur l'effet de serre. Version 1.2., Institut de l'Elevage, Paris, France, 156p.

Gaujour E., Amiaud B., Mignolet C., Plantureux S., 2012. Factors and processes affecting plant biodiversity in permanent grasslands. A review. Agron. Sustainable Dev., 32, 133-160.

Hansen M.N., Sommer S.G., Henriksen K., 2010. Methane emission from livestock manure-effects of storage conditions and climate. In: Sustainable Agriculture, vol. 2, Springer, $991 \mathrm{p}$.

Henrikson M., Flysjö A., Cederberg C., Swensson C., 2011. Variation in carbon footprint of milk due to management differences between Swedish dairy farms. Animal, 5, published online, doi:10.1017/S17517311110 00437.

Herzog F., Steiner B., Bailey D., Baudry J., Billeter R., Bukacek R., de Blust G., de Cock
R., Dirksen J., Dormann C.F., de Filippi R., Frossard E., Liira J., Schmidt T., Stockli R., Thenail C., van Wingerden W., Bugter R., 2006. Assessing the intensity of temperate European agriculture at the landscape scale. Eur. J. Agron., 24, 165-181.

Honnay O., Adriaens D., Coart E., Jacquemyn H., Roldan-Ruiz I., 2007. Genetic diversity within and between remnant populations of the endangered calcareous grassland plant Globularia bisnagarica L. Conserv. Genet., 8, 293-303.

Hutton S.A., Giller P.S., 2003. The effects of the intensification of agriculture on northern temperate dung beetle communities. J. Appl. Ecol., 40, 994-1007.

Isselstein J., Jeangros B., Pavlu V., 2005. Agronomic aspects of biodiversity targeted management of temperate grasslands in Europe - a review. Agron. Res., 3, 139-151.

Jan P., Dux D., Lips M., Alig M., Dumondel M., 2011. On the link between economic and environmental performance of Swiss dairy farms of the alpine area. LCA for Agriculture, 17, 706-719.

Jarvis S., Hutchings N., Brentrup F., Olesen J.E., Van de Hoek K.W., 2011. Nitrogen flows in farming systems across Europe. In: Sutton M.A., Howard C.M., Erisman J.W., Billen G., Bleeker A., Grennfelt P., Van Grinsven H., Grizzetti B. (Eds). Eur. Nitrogen Assessment. Sources, Effects and Policy Perspectives. Cambridge : Cambridge University Press, 211228.

Johnson K.A., Johnson D.E., 1995. Methane emissions from cattle. J. Anim. Sci., 73, 24832492.

Jules E.S., Shahani P., 2003. A broader ecological context to habitat fragmentation: why matrix habitat is more important than we thought. J. Veget. Sci., 14, 459-464.

Klumpp K., Fontaine S., Attard E., Gleixner G., Leroux X., Soussana J.F., 2009. How land use change shifts C-poor, porductive exosystems to C-rich, unproductive ecosystems and vice versa. J. Ecol., 91, 867-885.

Krauss J., Klein A.M., Steffan-Dewenter I., Tscharntke T., 2004. Effects of habitat area, isolation, and landscape diversity on plant species richness of calcareous grasslands. Biodiv. Conserv., 13, 1427-1439.

Laurent F., Vertès F., Farruggia A., Kerveillant P., 2000. Effets de la conduite de la prairie pâturée sur la lixiviation du nitrate. Propositions pour une maîtrise du risque à la parcelle. Fourrages, 164, 397-420.

Le Coeur D., Baudry J., Burel F., Thenail C., 2002. Why and how we should study field boundaries biodiversity in an agrarian landscape context. Agric., Ecosys. Environ., 89, 23-40.

Le Gall A., Vertès F., Pfimlin A., Chambaut H., Delaby L., Durand P., van der Werf H.M.G., Turpin N., Bras A., 2005. Nutrient management at farm scale. How to attain policy objectives in regions with intensive dairy farming ? - France. In: Nutrient management at farm scale. How to attain policy objectives with intensive dairy farming ? Bos J. Pflimlin A., Arts F., Vertès F. (Eds). First Workshop of the EGF Working Group "Dairy Farming Syst. Environ." 23-25 juin 2003, Quimper, France. 111-139.

Le Gall A., Beguin E., Dollé J.B., Manneville V., Pflimlin A., 2009. Nouveaux 
compromis techniques pour concilier les impératifs d'efficacité économique et environnementale des systèmes d'élevage herbivore. Fourrages, 198, 131-151.

Le Roux X., Barbault R., Baudry J., Bure F., Doussan I., Garnier E., Herzog F., Lavorel S., Lifran R., Roger-Estrade J., Sarthou J.P., Trommetter M., 2008. Agriculture et Biodiversité. Valoriser les synergies. Expertise scientifique collective, rapport, INRA, France, 728 p.

Lindborg R., Eriksson O., 2004. Historical landscape connectivity affects present plant species diversity. Ecology, 85, 1840-1845.

Loiseau P., Soussana J.F., Louault F., Delpy R., 2001. Soil N contributes to the oscillations of the white clover content in mixed swards under simulated grazing (Lolium perenne $L$, Trifolium repens L.). Grass Forage Sci, 56, 205-217.

McLaughlin A., Mineau P., 1995. The impact of agricultural practices on biodiversity. Agric., Ecosys. Environ. 55, 201-212.

Mc Mahon B.J., Helden A., Anderson A. Sheridan H., Kinsellac A., Purvis G., 2010. Interactions between livestock systems and biodiversity in South-East Ireland. Agric. Ecosys. Environ., 139, 232-238.

Martin C., Pomiès D., Ferlay A., Rochette Y., Martin B., Chilliard Y., Morgavi D.P., Doreau M., 2011. Methane output and rumen microbiota in dairy cows in response to longterm supplementation with linseed or rapeseed of grass silage or pasture based diets. Proc. N.Z. Soc. Anim. Prod., 71, 243-247.

Meschy F., 2010. Nutrition minérale des ruminants. Ed QUAE, Versailles, France, 208p.

Micol D., Hoch T., Agabriel J., 2003. Besoins protéiques et maîtrise des rejets azotés du bovin producteur de viande. Fourrages, 174, 231-242.

Mignolet C., Benoit M., Saint D., 1997. Système d'élevage et risque de pollution azoté. Construction D'un indicateur de risque et application dans la plaine des Vosges. INRA Prod. Anim., 10, 275-285.

Moreau P., Ruiz L., Vertès F., Baratte C., Delaby L., Faverdin P., Gascuel-Odoux C., Piquemal B., Ramat E., Salmon-Monviola J., Durand P., 2013. CASIMOD'N: an agrohydrological distributed model of catchmentscale nitrogen dynamics integrating farming system decisions, Agricult. Sys., 118C, 41-51

Mulder C., van Wezel A.P., van Wijnen H.J., 2005. Embedding soil quality in the planning and management of land use. Int. J. Biodiv. Sci. Management, 1, 1-8.

Muller S., Dutoit T., Alard. D., Grevilliot F., 1998. Restor. Ecol., 6, 94-101.

Paccard P., Capitain M, Farruggia A., 2003. Autonomie alimentaire et bilans des minéraux des élevages bovins laitiers selon les systèmes de production. Fourrages, 174, 243-257.

Paillat J.M., Robin P., Hassouna M., Callarec J. Toularastel P., 2005. Environmental assessment of composting pig slurry with wheat straw based on the Guernévez process. Proc. Conf. "Int. Workshop Green Pork Prod.", Paris, France.

Petersen S.O., Sommer S.G., 2011 Ammonia and nitrous oxide interactions: Roles of manure organic matter management. Anim. Feed Sci. Technol., 166-167, 503-513.

Peyraud J.L., Vérité R., Delaby L., 1995. Rejets azotés chez la vache laitière : effet du type d'alimentation et du niveau de production des animaux. Fourrages, 142, 131-144.

Peyraud J.L., Cellier P., Donnars C., Réchauchère O., 2012. Les flux d'azote liés aux élevages, réduire les pertes, rétablir les équilibres. Expertise scientifique collective, synthèse du rapport, $72 \mathrm{p}$

Plantureux S., 1996. Biodiversity, type of soil and management intensity of permanent pastures of Plateau Lorrain. Acta Bot. Gallica, 143, 339-348

Pykala J., Luotoa J., Heikkinena R. Kontulab P.T., 2005. Lant species richness and persistence of rare plants in abandoned seminatural grasslands in northern Europe. Basic Appl. Ecol., 6, 27, 25-33.

Raison C., Chambaut H., Le Gall A., Pflimlin A., 2008. Impact du système fourrager sur la qualité des eaux. Enseignements issus du projet Green Dairy. Fourrages, 193, 3-18.

Reeder J.D., Schuman G.E., 2002. Influence of livestock grazing on $\mathrm{C}$ sequestration in semi-arid mixed-grass and short-grass rangelands. Environ. Pollut., 116, 457-463.

Sauvant D., Giger-Reverdin S., Serment A. Broudiscou L., 2011. Influences des régimes et de leur fermentation dans le rumen sur la production de méthane par les ruminants. INRA Prod. Anim., 24, 433-446.

Schils R.L.M., Verhegen A., Aarts H.F.M. Sebek L.B.J., 2005. A farm level approach to define successful mitigation strategies for GHG emissions from ruminant livestock systems. Nutr. Cycl. Agroecosys. 71, 163-175.

Schröder J.J., Aarts H.F.M., ten Berge H.F.M., van Keulen H., Neeteson J.J., 2003. An evaluation of whole-farm nitrogen balances and related indices for efficient nitrogen use Europ. J. Agron. 20, 33-44

Scimone M., Rook A.J., Garel J.P., Sahin N., 2007. Effects of livestock breed and stocking rate on sustainable grazing systems: 3 . Effects on vegetation diversity. Grass Forage Sci., 62, 172-184.

Simon J.C., Le Corre L., 1992. Le bilan apparent de l'azote à l'échelle de l'exploitation agricole : méthodologie, exemple de résultats. Fourrages, 129, 79-94.

Simon J.C., Grignani C., Jacquet A., Le Corre L., Pagès., 2000. Agronomie, 20, 175-195

Sjödin E., Bengtsson J., Ekbom B., 2008. The influence of grazing intensity and landscape composition on the diversity and abundance of flower-visiting insects. J. Appl. Ecol., 45, 763 772 .
Soussana J.F., Lüscher A., 2007. Temperate grasslands and global atmospheric change: a review. Grass Forage Sci., 62, 127-134.

Soussana J.F., Tallec T., Blanfort V., 2010 Mitigating the greenhouse gas balance of ruminant production systems through carbon sequestration in grasslands. Animal, 4, 334350 .

Sullivan T.P., Sullivan D.S., 2006. Plant and small mammal diversity in orchard versus noncrop habitats. Agric., Ecosys. Environ., 116, 235-243.

Suominen O., Danell K., 2006. Effects of large herbivores on other fauna. In large herbivore ecology, Ecosystem Dynamics and Conservation. Danell K., Bergström R. Duncan P., Pastor J. (Eds). Cambridge University Press, 383-412.

Van Buskirk J., Willi Y, 2004. Enhancement of farmland biodiversity within set-aside land. Conservation Biol., 18, 987-994.

Van der Werf H.M.G., Kanyarushoki C., Corson M.S., 2009. An operational method for the evaluation of resource use and environmental impacts of dairy farms by life cycle assessment. J. Environ. Manage., 90, 3643-3652.

Vellinga T., de Haan M.H.A., Schils R.L.M., Evers A., Van den Pol-van Dasselaar A., 2011. Implementation of GHG mitigation on intensive dairy farms: farmers preferences and variation in cost effectiveness. Livest. Sci., 137, 185-195.

Vérité R., Delaby L., 2000. Relation between nutrition, performances and nitrogen excretion in dairy cows, Ann. Zootech., 49, 217-230.

Vermorel M., Jouany J.P., Eugène M. Sauvant D., Noblet J., Dourmad J.Y., 2008. Evaluation quantitative des émissions de méthane entérique par les animaux d'élevage en 2007 en France. INRA Prod. Anim., 21, 403-418.

Vertès F., Simon J.C., Le Corre L., Decau M.L., 1997. Les flux d'azote au pâturage. II Etude des flux et de leurs effets sur le lessivage. Fourrages, 151, 263-280.

Vertès F., Simon J.C., Laurent F., Besnard A., 2007. Prairies et qualité de l'eau. Evaluation des risques de lixiviation d'azote et optimisation des pratiques. Fourrages, 192, 423-440.

Vertès F., Benoit M., Dorioz J.M., 2010. Couverts herbacés pérennes et enjeux environnementaux (en particulier eutrophisation): atouts et limites. Fourrages, 102, 83-94.

Webb J., Sommer S.G., Kupper T., Groenestein K., Hutchings N.J., EuricheMenden B., Rodhe L., Misselbrook T.H., Amon B., 2012. Emissions of ammonia, nitrous oxide and methane during the management of solid manures. In: Agroecology and Strategies for Climate Change. Lichtfouse E. (Ed). 8, 67-107.

Woodward S.L., Waghorn G.C., Thomson N.A., 2006. Supplementing dairy cows with oils to improve performance and reduce methane - does it work? Proc. N.Z. Soc. Anim. Prod., 66, 176-181. 


\title{
Résumé
}

La préservation de l'environnement est actuellement au cœur des débats dans l'évolution des systèmes de production laitière. Le défi des années futures réside dans la fourniture de produits laitiers tout en assurant les performances sociales, économiques et environnementales, c'est-à-dire la durabilité des systèmes de production. En matière d'environnement, une attention toute particulière doit être portée à la limitation des risques de pollution vers l'eau, vers l'air et à la préservation de la biodiversité. Les systèmes d'élevage herbivore français, largement basés sur la prairie, présentent pour cela un réel atout du fait du lien au sol et de la part importante de fourrages dans l'alimentation du troupeau. Peu liée aux caractéristiques structurelles, l'efficience environnementale passe notamment par l'optimisation du recyclage du carbone et de l'azote au sein des systèmes de production. Alors que la variabilité des impacts environnementaux entre exploitations de systèmes différents (plaine vs montagne) est faible, une forte variabilité est observée entre exploitations d'un même système. Ceci démontre que les pratiques efficientes sur le plan technique, relatives à la gestion optimale des effectifs animaux, à une sobriété énergétique, etc. ont des incidences environnementales et économiques favorables. C'est pourquoi, une partie significative des exploitations d'élevage d'herbivores françaises devrait pouvoir être considérée à hautes performances environnementales et économiques sans remise en cause lourde du système de production. Par ailleurs l'élevage laitier, qui intervient pour réduire les pertes vers l'air et vers l'eau, est garant du stockage de carbone et de la biodiversité. Au-delà de la production de lait et de viande, l'élevage laitier joue dès lors un rôle majeur dans la préservation de l'environnement.

\begin{abstract}
Environmental impact of French dairy cattle livestock farming systems

Environmental issues are now at the heart of the debates concerning the evolution of dairy production systems. The challenge of the next few years lies in supplying dairy products while insuring social, economic and environmental performances, to improve sustainability of production systems. Regarding the environment, particular attention must be paid to limiting the risks of pollution towards water, air and the conservation of biodiversity. The French ruminant breeding system, widely based on grasslands, presents a real asset due to the link to the soil and from the large part of forage in the herd's diet. The environmental efficiency, which is not directly connected to the structural characteristics, occurs in particular by the optimization of carbon and nitrogen recycling within the production systems. While the variability of the environmental burdens between farms of different systems (plain vs mountain) is low, a strong variability is observed between farms of the same system. This demonstrates that the practices, which are efficient in terms of optimising animal management and low energy consumption..., have positive environmental and economic impacts. Therefore, a significant part of the French ruminant farms should be considered as having high environmental and economic performance without disrupting the current production systems. Finally dairy farming, which acts to reduce the carbon and nitrogen losses to the air and water, promotes carbon storage and biodiversity. Beyond the production of milk and meat, dairy farming therefore plays a major role in the preservation of the environment.
\end{abstract}

DOllé J.-B., DElabY L., PlantureuX S., MOREAU S., AMiAUD B., CHARPIOT A., MANNEVILLE V., CHANSEAUME A., CHAMBAUT H., LE GALL A., 2013. Impact environnemental des systèmes bovins laitiers français. In : Numéro spécial, La vache et le lait. Faverdin P., Leroux C., Baumont R. (Eds). INRA Prod. Anim., 26, 2, $207-220$. 